\title{
Traffic Adaptation and Energy Efficiency for Small Cell Networks with Dynamic TDD
}

\author{
Hongguang Sun, Member, IEEE, Min Sheng, Senior Member, IEEE, Matthias Wildemeersch, Member, IEEE, \\ Tony Q. S. Quek, Senior Member, IEEE, Jiandong Li, Senior Member, IEEE
}

\begin{abstract}
The traffic in current wireless networks exhibits large variations in uplink (UL) and downlink (DL), which brings huge challenges to network operators in efficiently allocating radio resources. Dynamic time-division duplex (TDD) is considered as a promising scheme that is able to adjust the resource allocation to the instantaneous UL and DL traffic conditions, also known as traffic adaptation. In this work, we study how traffic adaptation and energy harvesting can improve the energy efficiency (EE) in multi-antenna small cell networks operating dynamic TDD. Given the queue length distribution of small cell access points (SAPs) and mobile users (MUs), we derive the optimal UL/DL configuration to minimize the service time of a typical small cell, and show that the UL/DL configuration that minimizes the service time also results in an optimal network EE, but does not necessarily achieve the optimal EE for SAP or MU individually. To further enhance the network EE, we provide SAPs with energy harvesting capabilities, and model the status of harvested energy at each SAP using a Markov chain. We derive the availability of the rechargeable battery under several battery utilization strategies, and observe that energy harvesting can significantly improve the network $\mathrm{EE}$ in the low traffic load regime. In summary, the proposed analytical framework allows us to elucidate the relationship between traffic adaptation and network EE in future dense networks with dynamic TDD. With this work, we quantify the potential benefits of traffic adaptation and energy harvesting in terms of service time and EE.
\end{abstract}

Index Terms-Traffic adaptation, dynamic time-division duplex, small cell networks, energy harvesting, energy efficiency, multi-antenna systems, service time, Poisson point process

\section{INTRODUCTION}

In recent years, the increasing popularity of data hungry applications, driven by the explosive growth of smart phones, have posed great challenges to achieve the required data rates [1]. To meet such stringent capacity demand, two significant technological advances have been proposed: deploying ultradense small cell networks to increase the spatial reuse gain [2], and equipping base stations with multiple antennas to increase

This work was supported in part by the National Natural Science Foundation of China under Grant 61231008, Grant 61301176, and Grant 91338114, in part by the 111 Project (B08038), in part by the National S\&T Major Project (2015ZX03004004), in part by the A*STAR SERC under Grant 1224104048 , in part by the SUTD-ZJU Research Collaboration under Grant SUTD-ZJU/RES/01/2014, and in part by the MOE ARF Tier 2 under Grant MOE2015-T2-2-104.

H. Sun, M. Sheng (corresponding author) and J. Li are with the State Key Laboratory of Integrated Service Networks, Institute of Information Science, Xidian University, Xi'an, Shaanxi, 710071, China. (email: hgsun@xidian.edu.cn, msheng@mail.xidian.edu.cn, jdli@mail.xidian.edu.cn).

Matthias Wildemeersch is with the International Institute for Applied Systems Analysis, Laxenburg, Austria. (e-mail: wildemee@iiasa.ac.at).

T. Q. S. Quek is with the Singapore University of Technology and Design and the Institute for Infocomm Research, Singapore. (e-mail: tonyquek@sutd.edu.sg). the spectrum efficiency [3]. The emergence of these two concepts will accelerate the transformation of cellular network architecture to heterogenous networks consisting of diverse low-power small cell access points (SAPs) equipped with multiple antennas overlaid within the conventional macrocell network [4]. With such heterogeneity in cellular network architecture and the irregularity in base station locations, new cellular network models have emerged recently. Stochastic geometry has been shown to be a powerful tool in capturing the randomness of network nodes by using spatial point processes [5]-[7]. By modeling the locations of base stations with the Poisson point process (PPP), the performance evaluation of a two-tier downlink (DL) heterogeneous network is considered in [8], [9] and then generalized to a K-tier DL network in [10], [11].

Meanwhile, the massive deployment of small cells and the dramatic increase of multimedia applications result in asymmetric and dynamically changing uplink (UL) and DL traffic which varies significantly with time and between cells. Time-division duplex (TDD) systems have the capability to accommodate the changing traffic loads by varying the percentage of subframes dedicated to UL and DL transmissions, referred to as the traffic adaptation [12]. ${ }^{1}$ To adapt to the instantaneous traffic load, dynamic TDD with cell-specific UL/DL configuration is now under consideration in small cell networks by the Third Generation Partnership Project (3GPP) [13], [14]. Particularly, eight network scenarios have been considered in Release 11, where multiple picocells deployed homogeneously or coexisted with multiple macrocells (on the same or adjacent carrier frequency), can dynamically adjust their UL/DL configurations. Furthermore, three of the eight deployment scenarios have been analyzed in Release 12 , where considerable enhancement in network throughput was observed with dynamic TDD. However, dynamic TDD gives rise to two new types of cross-link interference, namely, mobile user-to-mobile user (UL-to-DL) interference and base station-to-base station (DL-to-UL) interference [15], when the neighboring SAPs operate on opposite modes.

The advantage of dynamic TDD over semi-static TDD in packet throughput has been evaluated based on both simulations [16] and theoretical analysis [15], [17] in single antenna small cell systems. Using the machinery of stochastic geometry, analytical expressions have been derived for DL and

\footnotetext{
${ }^{1}$ In TDD mode, a radio frame includes a number of subframes with each subframe denoting the unit time for one DL or UL transmission. For instance, in the TD-LTE (Long Term Evolution), one radio frame consists of ten subframes with the duration of one subframe being $1 \mathrm{~ms}$.
} 
UL coverage probabilities with dynamic TDD in single-tier small cell networks [18] and two-tier heterogeneous networks [19]. Particularly, in the full-buffer and fully-loaded network scenario, the per-tier UL/DL configuration is optimized in terms of the DL and UL coverage probabilities in [19]. However, the asymmetric UL and DL traffic loads are not captured, and the relationship between service time and optimal UL/DL configuration is not investigated.

On the other hand, green communications are drawing more and more attention on a global scale. From the ecological perspective, the information and communication technology (ICT) industry is believed to be responsible for almost $4.5 \%$ of the global greenhouse gas emissions by 2020 [20]. From the economic perspective, the rising energy consumption of mobile networks increases the energy costs and the operational expenditures (OPEX) of the providers. It is therefore highly important to improve the energy efficiency (EE) in future networks. To enhance the EE of the network, several solutions have been proposed, such as sleeping strategies [21]-[23] and energy harvesting techniques [24]-[26]. Specifically, with energy harvesting capabilities, base stations can harvest solar, wind, or kinetic energy from the environment [27], and thus reduce the use of conventional energy and the carbon footprint. Under the full-buffer assumption, optimal transmission strategies have been designed to adapt to the given energy arrival process in the single-antenna point-to-point link [28], broadcasting channel [29], and heterogeneous networks [24]. Specifically, in [24], a K-tier DL energy harvesting heterogeneous cellular network is considered where the authors study the availability region of the network and its impacts on the network coverage probability. To relax the full-buffer assumption, the data arrivals are explicitly incorporated by considering an additional data queue at each transmitter for a single-antenna isolated link [30] and a broadcasting link [31] with the objective to minimize the packet transmission delay. Most prior work concerns self-powered base stations and mainly focuses on the DL case with full-buffer assumption, while the effect of energy harvesting and dynamic TDD with traffic-aware model on EE has not been investigated. Such a network setting is of high practical relevance and leads to important research questions, for which the solution will shed light on the relationship between traffic adaptation, service time, and EE.

In this work, we consider traffic adaptation within an energy harvesting small cell network operating dynamic TDD, where each energy harvesting SAP is equipped with multiple antennas and operates spatial division multiple access (SDMA). All energy harvesting SAPs are connected to the power grid to ensure energy supply continuity, and preferentially consume the harvested energy to serve the UL or DL data streams. We consider a traffic-aware model and optimize the UL/DL configuration based on the UL and DL data queue length distribution. By modeling the rechargeable battery dynamics of an SAP as a finite state discrete-time Markov chain (DTMC), we derive the availability of the rechargeable battery and the EE of the typical cell. Our main contributions are listed as follows:

- We abandon the full-buffer assumption and assume a given queue length distribution for UL and DL. We derive the optimal UL/DL configuration that minimizes the service time of the typical small cell, and quantify the effect of UL/DL traffic loads on the network performance in terms of coverage probability, service time, and EE.

- We propose several battery utilization strategies and show that energy harvesting can improve the EE of the typical small cell substantially in the low traffic load regime.

- We study the relationship between traffic adaptation and EE. Our results demonstrate that the optimal UL/DL configuration that minimizes the service time also leads to the optimal network EE, while this conclusion does not hold for the EE of SAPs and mobile users (MUs) individually.

- We evaluate the effect of signal-to-interference-plus-noise ratio (SINR) threshold on the service time and network $\mathrm{EE}$, and observe that under realistic conditions the optimal setting of the SINR threshold can lead to a reduction of $60 \%$ of the service time and an improvement of $230 \%$ of the network EE.

The rest of the paper is organized as follows. In Section II, the system model is presented. In Section III, the power consumption model and the energy harvesting model are presented. In Section IV, the coverage probability and EE is derived. In Section V, the analytical results are validated via simulations and the effects of key parameters are discussed. Conclusions are given in Section VI.

\section{System Model}

\section{A. Network model}

We consider a small cell energy harvesting network, where the spatial locations of SAPs follow a homogeneous PPP $\tilde{\Phi}_{\mathrm{S}}$ with intensity $\lambda_{\mathrm{s}}$. Each SAP is equipped with an energy harvesting module and a rechargeable battery to store the harvested energy. Considering the limitations of energy harvesting, the SAPs are connected to the power grid to ensure energy continuity. The spatial locations of MUs, $\tilde{\Phi}_{\mathrm{u}}$, are modeled as a Matern cluster point process (MCPP) with intensity $\lambda_{\mathrm{u}}=N \lambda_{\mathrm{s}}$ [5]. Specifically, the SAPs constitute the clusters' parent process where each SAP is located at the center of a cluster with radius $R_{\mathrm{cl}}$. Within each cluster, $N$ daughter points representing MUs are uniformly distributed and connect to the SAP located at the cluster center. We consider that each SAP only serves the $N$ subscribed MUs. When an SAP is requested by its subscribed MUs, it preferentially consumes the harvested energy stored in the rechargeable battery.

Each SAP is equipped with $M$ antennas, while each MU has a single antenna. ${ }^{2}$ All small cells operate the dynamic TDD scheme, where a small cell configures flexibly in DL or UL mode according to the instantaneous traffic load within the cell. The transmission mode selection for small cells is modeled by independent Bernoulli random variables (r.v.'s), such that each small cell is configured in DL and UL mode with probabilities $q_{\mathrm{D}}$ and $1-q_{\mathrm{D}}$, respectively. In particular,

\footnotetext{
${ }^{2}$ By extending the single-tier model in this work into a multi-tier setting, we can model the scenario that the SAPs in different tiers are equipped with different number of antennas, similar to that considered in [10].
} 


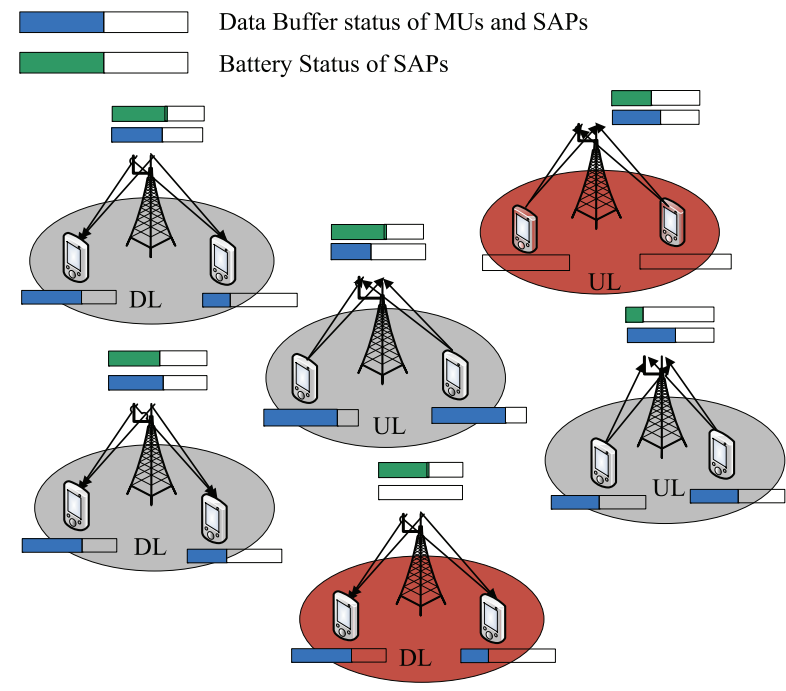

Figure 1. An illustration of two-antenna energy harvesting small cells with dynamic TDD scheme, where each SAP maintains two queues, one for data and the other for the harvested energy. An SAP or MU enters into sleeping mode when the DL or UL queue is empty.

we consider a dynamic TDD scheme without coordination of the patterns of UL and DL subframes across the small cells. Without coordination, it is impractical to have an exact model of the set of DL SAPs and UL MUs in each subframe. We therefore apply the independent Bernoulli approach which satisfies the UL/DL configuration $q_{\mathrm{D}}$ on average. The UL/DL configuration, denoted by the multiplexing probability $q_{\mathrm{D}}$, is not only determined by the UL and DL traffic conditions, but also affected by the interference from neighboring cells [32]. An illustration of the system model is shown in Fig. 1.

\section{B. Traffic load model}

In the following, we abandon the full-buffer condition typically assumed in prior work and propose a stylized trafficaware resource allocation model that can capture the general trend and provide relevant insights related to traffic adaptation. We assume that both the SAP and MU maintain an infinite buffer to accommodate the incoming packets. We define the marked point processes of MUs and SAPs where the marker indicates the buffer status, i.e., the number of packets stored in the buffer. We assume that the packet size $\varsigma$ [in bits] is fixed, and thus, the queue length can be utilized to represent the amount of traffic. For each SAP, we have $\hat{\Phi}_{\mathrm{s}}=\left\{\left(\mathbf{x}, \mathbf{b}_{\mathbf{s}, \mathbf{x}}\right) \mid \mathbf{x} \in \tilde{\Phi}_{\mathrm{s}}, \mathbf{b}_{\mathbf{s}, \mathbf{x}} \sim \mathbf{f}_{W}(w)\right\}$ with $\mathbf{b}_{\mathbf{s}, \mathbf{x}}$ indicating the queue length vector of an SAP located at $\mathrm{x}$ to all its associated $N$ MUs. Specifically, we define $\mathbf{b}_{\mathbf{s}, \mathbf{x}}=\left\{B_{\mathbf{s}, i}\right\}_{i=1}^{N}$ and $\mathbf{f}_{W}(w)=\left\{f_{W_{i}}(w)\right\}_{i=1}^{N}$ with $B_{\mathbf{s}, i} \sim f_{W_{i}}(w)$ denoting that the DL queue length of the SAP for $i$ th associated MU follows the function $f_{W_{i}}(w)$. While for MUs, we have $\hat{\Phi}_{\mathrm{u}}=\left\{\left(\mathbf{y}, B_{\mathrm{u}, \mathbf{y}}\right) \mid \mathbf{y} \in \tilde{\Phi}_{\mathrm{u}}, B_{\mathrm{u}, \mathbf{y}} \sim f_{U}(u)\right\}$ with $B_{\mathrm{u}, \mathbf{y}}$ denoting the queue length of an MU located at $\mathbf{y}$. In the proposed model, the queue length of both SAPs and MUs follows the geometric distribution, which is consistent with the $\mathrm{M} / \mathrm{M} / 1$ model [33]. Note that in practical scenarios, events that occur in consecutive subframes are correlated [34]. In fact, the variation of queue length over consecutive subframes results in variations of the interference, which affects outage capacity and service rate. Due to the coupling between the service rate and the interference process, such an exact approach leads to extremely complicated expressions that give little insight. Therefore, we propose a stylized model that ignores these correlations, but enables us to get more insights on the main trends in traffic adaptation. In particular, we assume that the queue length distribution is independent of the SAP's and MU's locations, which allows to omit the position in the subscript. With the geometric distribution, we have $B_{\mathrm{s}} \sim$ $G\left(1-\rho_{\mathrm{s}}\right)$ and $B_{\mathrm{u}} \sim G\left(1-\rho_{\mathrm{u}}\right)$, where the parameters $1-\rho_{\mathrm{s}}$ and $1-\rho_{\mathrm{u}}$ denote the fraction of idle time of the SAP and $\mathrm{MU}$, respectively. The probability mass function of $B_{\mathrm{s}}$ and $B_{\mathrm{u}}$ is, respectively, given by

$$
\begin{aligned}
\operatorname{Pr}\left\{B_{\mathrm{s}}=k\right\} & =\left(1-\rho_{\mathrm{s}}\right) \rho_{\mathrm{s}}^{k}, k \in \mathbb{N}, \\
\operatorname{Pr}\left\{B_{\mathrm{u}}=k\right\} & =\left(1-\rho_{\mathrm{u}}\right) \rho_{\mathrm{u}}^{k}, k \in \mathbb{N} .
\end{aligned}
$$

According to the $\mathrm{M} / \mathrm{M} / 1$ model, we can write $\rho_{\mathrm{s}}=\frac{\Lambda_{\mathrm{s}}}{\mu_{\mathrm{s}}}$ $\left(\rho_{\mathrm{u}}=\frac{\Lambda_{\mathrm{u}}}{\mu_{\mathrm{u}}}\right)$ where $\Lambda_{\mathrm{s}}\left(\Lambda_{\mathrm{u}}\right)$ and $\mu_{\mathrm{s}}\left(\mu_{\mathrm{u}}\right)$ denote the packet arrival rate and service rate of an SAP (MU), respectively. Note that due to the dynamic TDD scheme, the service rates $\mu_{\mathrm{s}}$ and $\mu_{\mathrm{u}}$ are related to the UL/DL configuration $q_{\mathrm{D}}$. In particular, a larger $q_{\mathrm{D}}$ means a larger portion of time for the DL transmissions, which increases the overall DL service rate $\mu_{\mathrm{s}}$ over the whole radio frame. Opposite effects hold for UL transmissions. To reflect the effect of $q_{\mathrm{D}}$ on the service rate, we define $\mu_{\mathrm{s}}=\mu_{\mathrm{s}, \min }+\kappa_{\mathrm{s}} q_{\mathrm{D}}$ and $\mu_{\mathrm{u}}=\mu_{\mathrm{u}, \min }+\kappa_{\mathrm{u}}\left(1-q_{\mathrm{D}}\right)$ where $\kappa_{\mathrm{s}}, \kappa_{\mathrm{u}}>0$ are system parameters, $\mu_{\mathrm{s}, \min }, \mu_{\mathrm{u}, \text { min }}>0$ denote the minimum DL and UL rates achieved when $q_{\mathrm{D}} \rightarrow 0$ and $q_{\mathrm{D}} \rightarrow 1$, respectively. We define the service rate $\mu_{\mathrm{s}}$ as a linear function of $q_{\mathrm{D}}$, as we assume that the DL service rate increases linearly with the allocated fraction of time. The same holds for the definition of $\mu_{\mathrm{u}}$. With the above definition, we have $\rho_{\mathrm{s}}=\frac{\Lambda_{\mathrm{s}}}{\mu_{\mathrm{s}, \min }+\kappa_{\mathrm{s}} q_{\mathrm{D}}}$ and $\rho_{\mathrm{u}}=\frac{\Lambda_{\mathrm{u}}}{\mu_{\mathrm{u}, \min }+\kappa_{\mathrm{u}}\left(1-q_{\mathrm{D}}\right)}$. For the queues to be stable and the network to achieve a stationary distribution, we require $\rho_{\mathrm{s}}<1$ and $\rho_{\mathrm{u}}<1$, which leads to $\Lambda_{\mathrm{s}}<\mu_{\mathrm{s}, \min }+\kappa_{\mathrm{s}} q_{\mathrm{D}}$ and $\Lambda_{\mathrm{u}}<\mu_{\mathrm{u}, \min }+\kappa_{\mathrm{u}}\left(1-q_{\mathrm{D}}\right){ }^{3}$ The condition $\mu_{\mathrm{s}, \min }, \mu_{\mathrm{u}, \min }>0$ is due to a technical reason to ensure the queue stability, i.e., $\rho_{\mathrm{s}}, \rho_{\mathrm{u}}<1$ within all the range of $q_{\mathrm{D}}$. When the network is in the stationary state, the probability that the queue length is empty for a random SAP and $\mathrm{MU}$ is, respectively, given by

$$
\begin{aligned}
& p_{\mathrm{s}, \text { void }}=\operatorname{Pr}\left\{B_{\mathrm{s}}=0\right\}=1-\frac{\Lambda_{\mathrm{s}}}{\mu_{\mathrm{s}, \text { min }}+\kappa_{\mathrm{s}} q_{\mathrm{D}}}, \\
& p_{\mathrm{u}, \text { void }}=\operatorname{Pr}\left\{B_{\mathrm{u}}=0\right\}=1-\frac{\Lambda_{\mathrm{u}}}{\mu_{\mathrm{u}, \min }+\kappa_{\mathrm{u}}\left(1-q_{\mathrm{D}}\right)} .
\end{aligned}
$$

We observe that $q_{\mathrm{D}}$ affects the void probabilities $p_{\mathrm{s} \text {,void }}$ and $p_{\mathrm{u}, \mathrm{void}}$ which change the active intensities of DL SAPs and UL MUs, respectively. The expectation of the queue length of

\footnotetext{
${ }^{3}$ The parameters $\kappa_{\mathrm{S}} \in(0,1)$ and $\kappa_{\mathrm{u}} \in(0,1)$ are used to reflect the effect of $q_{\mathrm{D}}$ on $\mu_{\mathrm{S}}$ and $\mu_{\mathrm{u}}$, and different values of these parameters will not change the overall conclusions of our work.
} 


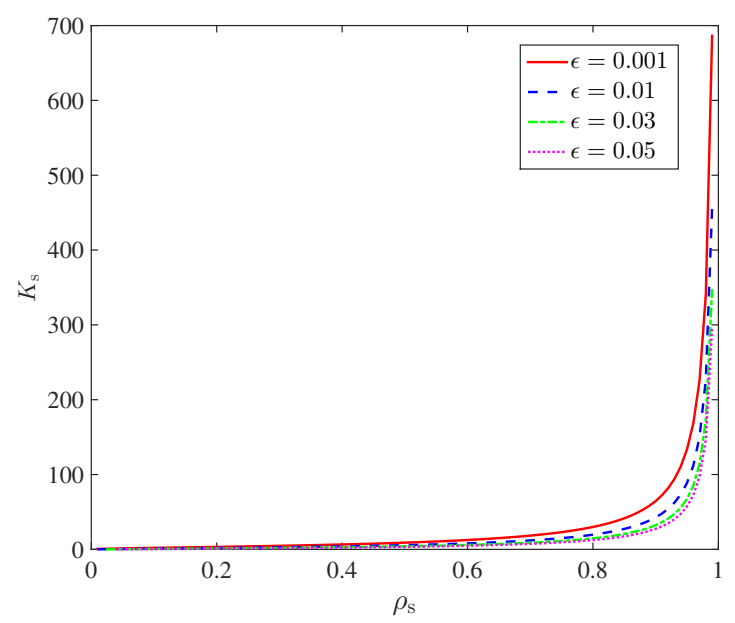

Figure 2. Minimum required buffer size $K_{\mathrm{s}}$ as a function of $\rho_{\mathrm{s}}$ for different packet dropping probability threshold $\epsilon$.

a random SAP and an MU is then given by

$$
\begin{aligned}
\mathbb{E}\left[B_{\mathrm{s}}\right] & =\frac{\rho_{\mathrm{s}}}{1-\rho_{\mathrm{s}}}=\frac{\Lambda_{\mathrm{s}}}{\mu_{\mathrm{s}, \min }+\kappa_{\mathrm{s}} q_{\mathrm{D}}-\Lambda_{\mathrm{s}}}, \\
\mathbb{E}\left[B_{\mathrm{u}}\right] & =\frac{\rho_{\mathrm{u}}}{1-\rho_{\mathrm{u}}}=\frac{\Lambda_{\mathrm{u}}}{\mu_{\mathrm{u}, \min }+\kappa_{\mathrm{u}}\left(1-q_{\mathrm{D}}\right)-\Lambda_{\mathrm{u}}} .
\end{aligned}
$$

Denote the spatial locations of active SAPs in DL mode and active MUs in UL mode as $\Phi_{\mathrm{s}}^{\mathrm{D}} \sim \operatorname{PPP}\left(\lambda_{\mathrm{s}, \mathrm{D}}\right)$ and $\Phi_{\mathrm{u}}^{\mathrm{t}}$. According to the thinning property of PPP, we have $\lambda_{\mathrm{s}, \mathrm{D}}=$ $q_{\mathrm{D}} \lambda_{\mathrm{s}}\left(1-p_{\mathrm{s}, \text { void }}\right) .{ }^{4}$ We also make an independent thinning to the UL MUs, such that the intensity of active MUs is given by $\lambda_{\mathrm{u}, \mathrm{t}} \backsim\left(1-q_{\mathrm{D}}\right) N_{\mathrm{a}} \lambda_{\mathrm{s}}$ where $N_{\mathrm{a}} \triangleq\left\lceil N\left(1-p_{\mathrm{u}, \text { void }}\right)\right\rceil$ denotes the number of active MUs associated with each UL SAP. ${ }^{5}$

Remark 1. The infinite buffer assumption results from a balanced tradeoff between realistic modeling and tractability, and whether the hypothesis is realistic depends on the traffic load and the actual buffer size. The use of a finite buffer leads potentially to dropped packets. To evaluate the impact of buffer size on the packet dropping probability, we define the buffer size of an SAP and an MU as $K_{\mathrm{s}}$ and $K_{\mathrm{u}}$. In the following, we take the DL case as an example, but the results are also applicable to the UL case. Let $\mathbb{P}_{\text {drop }}$ denote the packet dropping probability of a DL packet, which can be derived as

$$
\mathbb{P}_{\text {drop }}=\operatorname{Pr}\left\{B_{\mathrm{s}}>K_{\mathrm{s}}\right\}=1-\sum_{k=0}^{K_{\mathrm{s}}}\left(1-\rho_{\mathrm{s}}\right) \rho_{\mathrm{s}}^{k}=\rho_{\mathrm{s}}^{K_{\mathrm{s}}+1} .
$$

To evaluate the error caused by the infinite buffer assumption, we define a packet dropping probability threshold $\epsilon$. For realistic values of $\epsilon$, the buffer size $K_{\mathrm{s}}$ can be approximated to be infinite if $\mathbb{P}_{\text {drop }} \leq \epsilon$, and solving for $K_{\mathrm{s}}$ yields

\footnotetext{
${ }^{4}$ Note that we assume that an active SAP in DL mode simultaneously serves all the $N$ MUs in the analysis. While this assumption is reasonable since even the number of MUs served by an active DL SAP is smaller than $N$, these MUs still occupy the total available bandwidth and consume all powers of the antennas, which is equivalent to serving $N$ users.

${ }^{5}$ It is worth noting that for technical reasons we approximate the number of active MUs to be an integer by employing the ceil operator.
}

$K_{\mathrm{s}} \geq\left(\log \epsilon / \log \rho_{s}\right)-1$. To include the constraint on the buffer size, a $\mathrm{M} / \mathrm{M} / 1 / K_{s}$ queueing model should be applied, where the void probabilities and the expectations of the queue length can be derived similarly to Eq. (3) to Eq. (6).

Figure 2 exhibits the minimum required buffer size $K_{\mathrm{s}}$ as a function of $\rho_{\mathrm{s}}$ for different packet dropping probability threshold $\epsilon$. We observe that the instability region where $\mathbb{P}_{\text {drop }}>\epsilon$, shrinks with increasing $\epsilon$. Specifically, in the worst case when the network is heavily loaded with $\rho_{\mathrm{s}}=0.99, K_{\mathrm{s}}=687$ is required to satisfy the very strict packet dropping probability threshold $\epsilon=0.001$. While in most general network scenarios with $\rho_{\mathrm{s}} \in(0,0.9]$, a finite buffer with size $K_{s}<65$ is enough to satisfy even the strict packet dropping probability constraint.

\section{Channel model and transmission scheme}

The channel model consists of pathloss and fading, where the pathloss function is given by $g(\|x\|)=\frac{1}{\|x\|^{\alpha}}$ with $\alpha>2$ the pathloss exponent. We consider the block fading model with each block the duration of one subframe. The analysis is conducted within one subchannel that is exposed to flat fading rather than frequency selective fading. The fading power from a transmitter to the typical receiver is assumed to be an independent and identically distributed (i.i.d.) exponential r.v. $h \sim \exp (1)$, which corresponds to the Rayleigh fading. We consider the SDMA scheme, where the total number of MUs $N$ served by each SAP does not surpass the number of antennas $M$, i.e., $N \leq M$. Thus, in each cluster, all the MUs can be simultaneously served by its associated SAP. We consider zero-forcing $(\mathrm{ZF})$ precoding at the DL SAPs, and ZF receiver at UL SAPs with perfect knowledge of the channel state information (CSI). ${ }^{6}$ The channel power gain between an SAP and an MU is different when the SAP acts as the serving SAP or interfering SAP in both DL and UL mode, which is discussed in more details in Section III.

\section{Traffic Adaptation}

An important advantage of dynamic TDD is traffic adaptation which refers to the adjustment of the fraction of time for UL and DL transmissions to adapt to the instantaneous UL and DL traffic loads. Adopting the appropriate UL/DL configuration $q_{\mathrm{D}}$ to match the instantaneous traffic demands in DL and UL leads to improved packet throughput, energy saving, and reduction of packet delay. In this work, we use the service time to illustrate the benefits of traffic adaptation, which is defined as follows.

Definition 1. (Service Time) Given the queue length of the tagged SAP (for the typical MU) $B_{\mathrm{s}}$ and the queue length of the typical MU $B_{\mathrm{u}}$, the DL (UL) service time $\tau_{\mathrm{t}}^{\mathrm{D}}\left(\tau_{\mathrm{t}}^{\mathrm{U}}\right)$ is defined as the required number of radio frames to successfully transmit all the DL (UL) packets, and the cell service time $\tau_{\mathrm{t}, \text { cell }}$ is defined as $\tau_{\mathrm{t}, \text { cell }}=\max \left\{\tau_{\mathrm{t}}^{\mathrm{D}}, \tau_{\mathrm{t}}^{\mathrm{U}}\right\}$.

\footnotetext{
${ }^{6} \mathrm{ZF}$ beam-forming with imperfect CSI can be incorporated in this framework by using tools from [35], [36]. Compared with other linear receivers, such as the minimum mean-square error (MMSE) receiver, the $\mathrm{ZF}$ receiver has low complexity and allows us to provide important system design guidelines.
} 
Table I

TABLE OF ABBREVIATIONS AND SYMBOLS

\begin{tabular}{|c|c|}
\hline Notation & Description \\
\hline 3GPP, TDD & Third Generation Partnership Project, time-division duplex \\
\hline UL, DL, SINR & Uplink, downlink, signal-to-interference-plus-noise ratio \\
\hline SAP, MU, r.v.'s & Small cell access point, mobile user, random variables \\
\hline PPP, MCPP & Poisson point process, Matern cluster point process \\
\hline ZF, CSI, ARQ & Zero-forcing, channel state information, automatic repeat-request \\
\hline EE, SDMA, OPEX & Energy efficiency, spatial division multiple access, operational expenditures \\
\hline CDF, PDF & Cumulative distribution function, probability density function \\
\hline DTMC, ICT & discrete-time Markov chain, information and communication technology \\
\hline$\tilde{\Phi}_{\mathrm{s}}, \tilde{\Phi}_{\mathrm{u}}$ & Point processes modeling the locations of total SAPs and MUs \\
\hline$\hat{\Phi}_{\mathrm{s}}, \hat{\Phi}_{\mathrm{u}}$ & $\begin{array}{l}\text { Marked point processes of SAPs and MUs with markers } B_{\mathrm{s}} \text { and } B_{\mathrm{u}} \\
\text { respectively denoting the queue length of an SAP and MU }\end{array}$ \\
\hline$\Phi_{\mathrm{s}}^{\mathrm{D}}, \Phi_{\mathrm{s}}^{\mathrm{U}}$ & Point processes modeling the locations of DL and UL SAPs \\
\hline$q_{\mathrm{D}}$ & Probability that an SAP operates on DL mode \\
\hline$\lambda_{\mathrm{s}}, \lambda_{\mathrm{u}}$ & Deployment intensities of SAPs, and MUs \\
\hline$P_{\mathrm{s}}, Q_{\mathrm{u}}$ & DL transmit power to each MU, transmit power of each MU \\
\hline$M, N$ & \# of antennas of each SAP, maximum \# of MUs served by each SAP \\
\hline$h_{0,0}^{\mathrm{D}}, h_{0,0}^{\mathrm{U}}$ & $\begin{array}{l}\text { Channel power of direct typical link in DL and UL mode with the distribution } \\
h_{0,0}^{\mathrm{D}} \sim \Gamma(M-N+1 ; 1), h_{0,0}^{\mathrm{U}} \sim \Gamma\left(M-N_{\mathrm{a}}+1 ; 1\right)\end{array}$ \\
\hline$g_{\mathbf{x}_{i}, \mathrm{SAP}}^{\mathrm{D}}, g_{\mathbf{x}_{i}, \mathrm{SAP}}^{\mathrm{U}}$ & $\begin{array}{l}\text { Channel power of the interfering link from a DL SAP located at } \mathbf{x}_{i} \\
\text { to the typical receiving MU and the tagged UL SAP with the } \\
\text { distribution } g_{\mathbf{x}_{i} \text { SAP }}^{\mathrm{D}}, g_{\mathbf{x}_{i} \text {, SAP }}^{\mathrm{U}} \sim \Gamma(N ; 1)\end{array}$ \\
\hline$g_{\mathrm{MU}}^{\mathrm{D}}, g_{\mathrm{MU}}^{\mathrm{U}}$ & $\begin{array}{l}\text { Channel power of the interfering link from a UL MU to the typical receiving } \\
\text { MU and the tagged UL SAP with the distribution } g_{\mathrm{MU}}^{\mathrm{D}}, g_{\mathrm{MU}}^{\mathrm{U}} \sim \operatorname{Exp}(1)\end{array}$ \\
\hline$\gamma_{\mathrm{D}}, \gamma_{\mathrm{U}}, R_{\mathrm{cl}}$ & DL and UL SINR thresholds, radius of each cluster \\
\hline$B_{\mathrm{s}}, B_{\mathrm{u}}$ & Queue length of a random SAP (for each MU), and a random MU \\
\hline$\Lambda_{\mathrm{s}}, \Lambda_{\mathrm{u}}$ & Packet arrival rate to a random SAP and a random MU \\
\hline$\mu_{\mathrm{s}, \min }, \mu_{\mathrm{u}, \min }$ & Minimum service time of a random SAP and a random MU \\
\hline$C_{\text {ene, }} K$ & Energy harvesting threshold, the rechargeable battery capacity \\
\hline
\end{tabular}

Given the queue length distribution of both SAPs and MUs, our objective is to minimize the service time of the typical small cell by setting an optimal UL/DL configuration $q_{\mathrm{D}}^{\star}$.

Remark 2. We consider the static case, where the locations of SAPs $\tilde{\Phi}_{\mathrm{s}}$ and MUs $\tilde{\Phi}_{\mathrm{u}}$ remain unchanged during the transmissions [37]. When the transmission starts, new arriving packets at the transmitter are not taken into account. We consider the stop-and-wait automatic repeat-request (ARQ) protocol without limit on the retransmission times, where the feedback channel is assumed to be error-free and delay-free [38]. Moreover, the transmissions in different subframes are assumed to be independent.

We consider constant bit-rate coding, and define $\gamma_{\mathrm{D}}$ and $\gamma_{\mathrm{U}}$ as the DL and UL SINR thresholds. A packet is said to be successfully transmitted if the received SINR is greater than the given threshold. Otherwise, the packet returns to the head of the queue awaiting retransmission in the subsequent subframes. The coverage probability in DL and UL mode is, respectively, given by

$$
\mathbb{P}_{\mathrm{D}}=\operatorname{Pr}\left(\operatorname{SINR}_{\mathrm{D}}>\gamma_{\mathrm{D}}\right), \quad \mathbb{P}_{\mathrm{U}}=\operatorname{Pr}\left(\operatorname{SINR}_{\mathrm{U}}>\gamma_{\mathrm{U}}\right) .
$$

Given the available bandwidth $W$, the target rate $\mathrm{R}_{\ell}$ is given by $\mathrm{R}_{\ell}=W \log _{2}\left(1+\gamma_{\ell}\right), \ell \in\{\mathrm{D}, \mathrm{U}\}$. By definition, the outage capacity of the typical link in mode $\ell$ is given by $\mathrm{R}_{\ell} \mathbb{P}_{\ell}, \ell \in$ $\{\mathrm{D}, \mathrm{U}\}$.

Define the duration of a radio frame as $T_{\mathrm{f}}=N_{\mathrm{sf}} \cdot \tau_{\mathrm{sf}}$ with $N_{\mathrm{sf}}$ and $\tau_{\mathrm{sf}}$ denoting the number of subframes within a radio frame and the duration of a subframe [in seconds]. The fraction of subframes dedicated to the DL traffic, i.e., the DL duty cycle, is derived as $q_{\mathrm{D}}=\frac{n_{\mathrm{d}}}{N_{\mathrm{sf}}}, n_{\mathrm{d}} \leq N_{\mathrm{sf}}$. Similarly, the UL duty cycle is given by $1-q_{\mathrm{D}}=\frac{N_{\mathrm{sf}}-n_{\mathrm{d}}}{N_{\mathrm{sf}}}$. In each subframe, we denote $1_{\left\{\mathrm{SINR}_{\mathrm{D}} \geq \gamma_{\mathrm{D}}\right\}}$ and $1_{\left\{\mathrm{SINR}_{\mathrm{U}} \geq \gamma_{\mathrm{U}}\right\}}$ as the transmission indicators for the DL and UL. Given $q_{\mathrm{D}}$ and the queue length distribution, the number of radio frames required to complete the DL and UL traffic are, respectively, derived as (8) and (9), at the top of next page, respectively. Note that in (8) and (9), (a) and (b) are derived by applying Jensen's inequality to the convex function $\frac{1}{x}, x>0$, and $q_{\mathrm{D}} T_{\mathrm{f}}$ and $\left(1-q_{\mathrm{D}}\right) T_{\mathrm{f}}$ in the denominator, respectively, denote the duration of DL and UL subframes within a radio frame. Thus, the service time of the typical cell is derived as

$$
\tau_{\mathrm{t}}=\max \left\{\tau_{\mathrm{t}}^{\mathrm{D}}, \tau_{\mathrm{t}}^{\mathrm{U}}\right\} .
$$

Obviously, the UL/DL configuration $q_{\mathrm{D}}$ has considerable effect on the service time of the small cell.

Define the duration of one subframe $\tau_{\mathrm{sf}}$ as the unit time, the average ratio of subframes required to successfully transmit the DL traffic to those required to successfully transmit the UL traffic is given by

$$
\Xi=\mathbb{E}\left[\frac{B_{\mathrm{s}} \varsigma / \tau_{\mathrm{sf}} \mathrm{R}_{\mathrm{D}} 1_{\left\{\mathrm{SINR}_{\mathrm{D}}>\gamma_{\mathrm{D}}\right\}}}{B_{\mathrm{u}} \varsigma / \tau_{\mathrm{sf}} \mathrm{R}_{\mathrm{U}} 1_{\left\{\mathrm{SINR}_{\mathrm{U}}>\gamma_{\mathrm{U}}\right\}}}\right] .
$$

To minimize the service time of the typical cell, $q_{\mathrm{D}}$ should be set to match the subframe ratio defined in (11), i.e., $\frac{q_{\mathrm{D}}}{1-q_{\mathrm{D}}}=\Xi$. Thus, we have the optimal UL/DL configuration $q_{\mathrm{D}}^{\star}$ given by

$$
q_{\mathrm{D}}^{\star}=\frac{\Xi}{\Xi+1} .
$$




$$
\begin{aligned}
\tau_{\mathrm{t}}^{\mathrm{D}} & =\mathbb{E}\left[\frac{B_{\mathrm{s}} \varsigma}{q_{\mathrm{D}} T_{\mathrm{f}} \mathrm{R}_{\mathrm{D}} 1_{\left\{\mathrm{SINR}_{\mathrm{D}}>\gamma_{\mathrm{D}}\right\}}}\right] \stackrel{(a)}{\geq} \frac{\varsigma}{q_{\mathrm{D}} T_{\mathrm{f}} \mathrm{R}_{\mathrm{D}}} \frac{\mathbb{E}\left[B_{\mathrm{s}}\right]}{\mathbb{E}\left[1_{\left\{\mathrm{SINR}_{\mathrm{D}}>\gamma_{\mathrm{D}}\right\}}\right]}=\frac{\Lambda_{\mathrm{s}} \varsigma}{q_{\mathrm{D}} T_{\mathrm{f}} \mathrm{R}_{\mathrm{D}} \mathbb{P}_{\mathrm{D}}\left(\mu_{\mathrm{s}, \min }+\kappa_{\mathrm{s}} q_{\mathrm{D}}-\Lambda_{\mathrm{s}}\right)} \\
\tau_{\mathrm{t}}^{\mathrm{U}} & =\mathbb{E}\left[\frac{B_{\mathrm{u}}}{\left(1-q_{\mathrm{D}}\right) T_{\mathrm{f}} \mathrm{R}_{\mathrm{U}} 1_{\left\{\mathrm{SINR}_{\mathrm{U}}>\gamma_{\mathrm{U}}\right\}}}\right] \stackrel{(b)}{\geq} \frac{\varsigma}{\left(1-q_{\mathrm{D}}\right) T_{\mathrm{f}} \mathrm{R}_{\mathrm{U}}} \frac{\Lambda_{\mathrm{u}} \varsigma}{\mathbb{E}\left[B_{\mathrm{u}}\right]}
\end{aligned}
$$

\section{Power Consumption And EnERgy Harvesting MODEL}

\section{A. Power consumption model}

In this section, we present the power consumption model for both SAPs and MUs. Considering the traffic loads, an SAP in DL mode or an MU in UL mode is active only when its queue is not empty. Otherwise, the DL SAP or the UL MU enters into the low-power sleeping mode, as such, in a given subframe, an SAP or an MU operates in one of the three modes: transmitting, receiving, or sleeping. By incorporating the effect of traffic load and differentiating the power consumption of SAPs from MUs in both DL and UL, the proposed power consumption model leads to a more accurate insight into the power consumption under realistic conditions in TDD networks.

In the DL subframe, the power consumption of an active SAP serving $n$ MUs is given by [39]

$$
P_{\mathrm{tr}, n}=\frac{n}{\eta_{\mathrm{s}}} P_{\mathrm{s}}+M P_{\mathrm{c}}+P_{0, \mathrm{D}}, n \geq 1,
$$

where $P_{\mathrm{S}}$ is the transmit power of an SAP to each MU, $\eta_{\mathrm{s}} \in$ $(0,1]$ is the efficiency of the power amplifier, $M P_{c}$ denotes the dynamic circuit power consumption which is proportional to the antenna number $M$, and $P_{0, \mathrm{D}}$ is the static power of an active SAP in DL mode, which includes the cooling power and the baseband processing power. The power consumption for a receiving $\mathrm{MU}$ is given by $Q_{\mathrm{rec}}=Q_{\mathrm{r}}+Q_{\mathrm{dec}}+Q_{0, \mathrm{D}}$, where $Q_{\mathrm{r}}, Q_{\mathrm{dec}}$, and $Q_{0, \mathrm{D}}$, respectively, denote the average power consumption of each MU's receiver antenna unit, the power consumed for decoding the desired DL signal, and the static power for an MU in DL mode.

In the UL subframe, the power consumption of an active UL SAP serving $n$ MUs is given by

$$
P_{\mathrm{rec}, n}=n P_{\mathrm{dec}}+M P_{\mathrm{r}}+P_{\mathrm{mud}}+P_{0, \mathrm{U}}, n \geq 1,
$$

where $P_{\text {dec }}$ is the power required to decode each MU's encoded information stream, $P_{\mathrm{r}}$ denotes the power consumption of each SAP's receiver antenna unit, $P_{\text {mud }}$ is the power consumption for the multiuser detection, and $P_{0, \mathrm{U}}$ is the static power of an active SAP in UL mode. The power consumed by each active $\mathrm{MU}$ in UL mode is modeled as $Q_{\mathrm{tr}}=\frac{1}{\eta_{\mathrm{u}}} Q_{\mathrm{u}}+Q_{\mathrm{c}}+Q_{0, \mathrm{U}}$, where $\eta_{\mathrm{u}} \in(0,1]$ represents the efficiency of the power amplifier of each $\mathrm{MU}, Q_{\mathrm{u}}, Q_{\mathrm{c}}$, and $Q_{0, \mathrm{U}}$ denote the transmit power, circuit power and static power for an active MU in UL mode, respectively. For the sleeping mode, the power consumption of an SAP and an MU is, respectively, given by $P_{\text {sleep }}$ and $Q_{\text {sleep }}$.

\section{B. Energy harvesting model}

Each SAP is equipped with a rechargeable battery with finite capabilities to accommodate the harvested energy. We assume that a rechargeable battery has $K+1$ levels with level 0 and level $K$ indicating an empty battery and a fully charged battery. Define $A(t)$ and $B(t)$ as the incoming energy and the battery energy level of an SAP at the beginning of subframe $t$. We model the energy arrival process $\{A(t)\}_{t=0}^{\infty}$ as an i.i.d. Bernoulli process with $\operatorname{Pr}\left\{A(t) \geq C_{\text {ene }}\right\}=\nu$, where $C_{\text {ene }}$ [in Joule] is the energy harvesting threshold to activate the energy harvesting circuit [40]. If $A(t) \geq C_{\text {ene }}$, the energy harvested within subframe $t$ is assumed to be $C_{\text {ene }}$ which is referred to as one energy unit. Otherwise, no energy is harvested. The energy arrival process is assumed to be independent for all SAPs [41] and can happen simultaneously with data transmissions. The sequence of battery energy levels $\{B(t)\}_{t=0}^{\infty}$ can be described by a finite state DTMC with the state space $\{0,1, \ldots, K\}$, where state $k$ means that the rechargeable battery has accumulated $k C_{\text {ene }}$ Joule energy at the beginning of subframe $t \geq 1$. We define the transition probability of the DTMC as $p_{k, m}=\operatorname{Pr}\{B(t)=m \mid B(t-1)=k\}$ with $k, m \leq K$. For the energy harvesting model, we have the following modeling assumptions.

Assumption 1 (Power consumption for each MU): Suppose that the MUs associated with the same SAP share all the power components as given in (13) (for DL) and (14) (for UL). The power consumptions for serving each $\mathrm{MU}$ in DL and UL are redefined as $\tilde{P}_{\mathrm{tr}, 1}=\frac{1}{N} P_{\mathrm{tr}, N}$ and $\tilde{P}_{\mathrm{rec}, 1}=\frac{1}{N} P_{\mathrm{rec}, N}$, where $N$ is the total number of MUs associated with each SAP.

Assumption 2 (Energy unit and battery capacity): Suppose that one energy unit $C_{\text {ene }}$ is enough to support one UL data stream, i.e., $C_{\text {ene }}=\tilde{P}_{\text {rec, } 1} T$ with $T$ denoting the duration of one subframe. Define $\omega=\left\lceil\frac{\tilde{P}_{\mathrm{tr}, 1}}{\tilde{P}_{\mathrm{rec}, 1}}\right\rceil$ and we have $\tilde{P}_{\mathrm{tr}, 1} T=$ $\omega C_{\text {ene }}$, where $\lceil\cdot\rceil$ is the ceiling operator. The energy consumption for serving $N$ DL and UL data streams are respectively given by $\tilde{P}_{\mathrm{tr}, \mathrm{N}} T=\omega N C_{\text {ene }}$ and $\tilde{P}_{\text {rec, } \mathrm{N}} T=N C_{\text {ene. }}$. We define the battery capacity as $K C_{\text {ene }}=c \omega N C_{\text {ene }}, c \in \mathbb{N}$, which means that a fully charged battery can support $N$ DL data streams for $c$ continuous subframes (or $N$ UL data streams for $c \omega$ subframes).

The above assumptions provide a feasible way to map the energy consumption of an SAP serving different number of data streams to the corresponding battery energy levels. This simplifies the analysis of the DTMC without losing insights from the energy harvesting technique. Keep $\tilde{P}_{\text {rec, } 1}$ unchanged, increasing $C_{\text {ene }}$ improves the availability of the rechargeable battery and enhances the EE. While a larger $\omega$ means an increasing power consumption on DL, which decreases the 
availability of the rechargeable battery and leads to a decrease on EE.

With the energy harvesting model, we are interested in the effect of different battery utilization strategies on EE. Specifically, we consider the following two strategies.

Strategy 1: The rechargeable battery is not activated until the harvested energy is enough to support $N$ data streams in either DL or UL mode. In other words, the battery is available for UL and DL transmissions when the energy level is no less than $N$ and $\omega N$ in the next subframe.

Strategy 2: The rechargeable battery is available for UL and DL transmissions as long as it has accumulated enough energy to support one data stream in either mode. In other words, the rechargeable battery is available for UL and DL transmissions when the battery energy level is no less than 1 and $\omega$ in the next subframe.

We define the probability $p_{\mathrm{D}, m}^{\text {avl }}$ and $p_{\mathrm{U}, m}^{\text {avl }}, m \in\{1,2\}$ as the availability of the rechargeable battery for DL and UL transmissions with Strategy $\mathrm{m}$. When the rechargeable battery is unavailable or the harvested energy is not enough to support all users, the SAP resorts to the back-up power grid. Letting $\boldsymbol{\pi}=\left\{\pi_{0}, \pi_{1}, \cdots, \pi_{K}\right\}$ denote the steady state probability vector, and in the steady state, we have $\boldsymbol{\pi} \mathbf{P}=\boldsymbol{\pi}$, where $\mathbf{P}=\left[p_{k, m}\right]_{(K+1) \times(K+1)}$ denotes the transition probability matrix. The stationary probabilities $\left\{\pi_{k}\right\}$ can be computed by solving $\boldsymbol{\pi} \mathbf{P}=\boldsymbol{\pi}$ under the constraint $\sum_{k=0}^{K} \pi_{k}=1$. In Lemma 1, we present the availability of the battery for DL and UL transmissions with different strategies.

Lemma 1. Given $\pi$, the availabilities of the battery for DL and UL transmissions under the two battery utilization strategies are respectively given by (15) and (16):

$$
\begin{aligned}
& p_{\mathrm{D}, 1}^{\mathrm{avl}}=\nu \pi_{\omega N-1}+\sum_{k=\omega N}^{K} \pi_{k}, \quad p_{\mathrm{U}, 1}^{\mathrm{avl}}=\nu \pi_{N-1}+\sum_{k=N}^{K} \pi_{k}, \\
& p_{\mathrm{D}, 2}^{\mathrm{avl}}=\nu \pi_{\omega-1}+\sum_{k=\omega}^{K} \pi_{k}, \quad p_{\mathrm{U}, 2}^{\mathrm{avl}}=\nu \pi_{0}+\sum_{k=1}^{K} \pi_{k},
\end{aligned}
$$

where we assume $K=c \omega N$ with $c, \omega, N \in \mathbb{N}$, and $\omega>N$.

Proof: Take DL as an example, with the stationary probabilities $\left\{\pi_{k}\right\}$, a battery is available as long as the harvested energy level in next subframe is no less than $\omega N$ and $\omega$ with Strategy 1 and Strategy 2. Thus, we have $p_{\mathrm{D}, 1}^{\text {avl }}=1-(1-\nu) \pi_{\omega N-1}$, and $p_{\mathrm{D}, 2}^{\text {avl }}=1-(1-\nu) \pi_{\omega-1}$. The final results are derived by reformulating the probabilities.

Remark 3. Although it is in general difficult to derive the stationary probabilities in closed-form, we can derive $\left\{\pi_{k}\right\}$ numerically by using standard software with transition probabilities given in Appendix A.

\section{Performance Analysis}

\section{A. Coverage probability}

In DL mode, the received signal for a typical MU located at the origin $\mathbf{0}$ is given by

$$
\begin{aligned}
y_{0}= & \sqrt{P_{\mathrm{s}}} r_{0}^{-\frac{\alpha}{2}} \mathbf{h}_{0,0}^{\dagger} \mathbf{w}_{0,0} s_{0}+\sum_{\mathbf{x}_{i} \in \Phi_{\mathrm{s} \backslash\{0\}}} \sqrt{P_{\mathrm{s}}}\left\|\mathbf{x}_{0, i}\right\|^{-\frac{\alpha}{2}} \\
& \cdot \mathbf{h}_{0, i}^{\dagger} \mathbf{W}_{i} \mathbf{s}_{i}+\sum_{\mathbf{z}_{j} \in \Phi_{\mathrm{u}}^{\mathrm{t}}} \sqrt{Q_{\mathrm{u}}}\left\|\mathbf{z}_{0, j}\right\|^{-\frac{\alpha}{2}} h_{0, j} u_{j}+n_{0},
\end{aligned}
$$

where $P_{\mathrm{s}}$ and $Q_{\mathrm{u}}$ are the transmit powers of SAP (to each MU) and $\mathrm{MU}, s_{0} \in \mathbb{C}$ is the normalized desired data symbol from the tagged SAP located at $\mathbf{x}_{0}, \mathbf{s}_{i} \in \mathbb{C}^{N \times 1}$ is the normalized transmit data symbol vectors of SAPs located at $\mathbf{x}_{i}, u_{j} \in \mathbb{C}$ is the normalized data symbol from the UL MU located at $\mathbf{z}_{j}$, and the additive Gaussian noise is given by $n_{0} \sim \mathcal{C N}\left(0, \sigma^{2}\right)$. The channel vector from the serving DL SAP located at $\mathbf{x}_{0}$ to the typical MU is denoted by $\mathbf{h}_{0,0} \in \mathbb{C}^{M \times 1}$ and that from the interfering DL SAP located at $\mathbf{x}_{i}$ is denoted by $\mathbf{h}_{0, i} \in \mathbb{C}^{M \times 1}$. For the UL MUs, the channel gain from $\mathbf{z}_{j}$ to the typical MU is denoted by $h_{0, j} \sim \mathcal{C N}(0,1)$. The vectors $\mathbf{h}_{0}, \mathbf{h}_{0, i}$ are assumed to have i.i.d. $\mathcal{C N}(0,1)$ entries, which are independent across DL SAPs, $\mathbf{W}_{i}=\left[\mathbf{w}_{i, k}\right]_{1 \leq k \leq N} \in \mathbb{C}^{M \times N}$ is the ZF precoding matrix set by the DL SAP located at $\mathbf{x}_{i}$.

From (17), the SINR at the typical MU associated with the DL tagged SAP is given by

$$
\mathrm{SINR}_{\mathrm{D}}=\frac{P_{\mathrm{s}} r_{0}^{-\alpha}\left\|\mathbf{h}_{0,0}^{\dagger} \mathbf{w}_{0,0}\right\|^{2}}{I_{\mathrm{D} \rightarrow \mathrm{D}}+I_{\mathrm{U} \rightarrow \mathrm{D}}+\sigma^{2}},
$$

where $I_{\mathrm{D} \rightarrow \mathrm{D}}$ and $I_{\mathrm{U} \rightarrow \mathrm{D}}$, respectively, denote the aggregate interference from DL active SAPs and UL MUs, given by

$$
\begin{aligned}
& I_{\mathrm{D} \rightarrow \mathrm{D}}=\sum_{\mathbf{x}_{i} \in \Phi_{\mathrm{s}}^{\mathrm{D}} \backslash\{0\}} P_{\mathrm{s}}\left\|\mathbf{x}_{0, i}\right\|^{-\alpha}\left|\mathbf{h}_{0, i}^{\dagger} \mathbf{W}_{i}\right|^{2}, \\
& I_{\mathrm{U} \rightarrow \mathrm{D}}=\sum_{\mathbf{z}_{j} \in \Phi_{\mathrm{u}}^{\mathrm{t}}} Q_{\mathrm{u}}\left\|\mathbf{z}_{0, j}\right\|^{-\alpha}\left|h_{0, j}\right|^{2} .
\end{aligned}
$$

Denote $\tilde{\mathbf{H}}_{i}=\left[\tilde{\mathbf{h}}_{0, i}, \ldots, \tilde{\mathbf{h}}_{k, i}, \ldots, \tilde{\mathbf{h}}_{N-1, i}\right]^{\dagger} \in \mathbb{C}^{N \times M}$ as the channel matrix between a DL interfering SAP and all the $N$ MUs associated with it. The direction of each vector channel is represented by $\tilde{\mathbf{h}}_{k, i} \triangleq \frac{\mathbf{h}_{k, i}}{\left\|\mathbf{h}_{k, i}\right\|}$, where $\|\cdot\|$ denotes the Euclidean norm. With ZF precoding, the columns of the precoding matrix $\mathbf{W}_{i}=\left[\mathbf{w}_{i, k}\right]_{1 \leq k \leq N} \in \mathbb{C}^{M \times N}$ are exactly the columns of the pseudo-inverse $\mathbf{H}_{i}^{\mathrm{H}} \triangleq \tilde{\mathbf{H}}_{i}^{\dagger}\left(\tilde{\mathbf{H}}_{i} \tilde{\mathbf{H}}_{i}^{\dagger}\right)^{-1} \in$ $\mathbb{C}^{M \times N}$, where $(.)^{\dagger}$ and $(.)^{\mathrm{H}}$ respectively denotes the conjugate transpose and the pseudo-inverse. When the queue length of the SAP is not empty, it simultaneously serves all the $N$ associated MUs and the desired channel power gain can be derived by $h_{0,0}^{\mathrm{D}}=\left\|\mathbf{h}_{0,0}^{\dagger} \mathbf{w}_{0,0}\right\|^{2} \sim \Gamma(M-N+1,1)$ [10]. The DL interference channel power gain is given by $g_{\mathbf{x}_{i}, \mathrm{SAP}}^{\mathrm{D}}=\left|\mathbf{h}_{0, i}^{\dagger} \mathbf{W}_{i}\right|^{2} \sim \Gamma(N, 1)$, and the UL interference channel power gain is derived as $g_{\mathrm{MU}}^{\mathrm{D}}=\left|h_{0, j}\right|^{2} \sim \operatorname{Exp}(1)$.

In UL mode, we assume that the typical UL MU is located at $z_{0}$, and the tagged SAP is located at the origin, $r_{0}=\left\|z_{0}\right\|$. The $M$-dimensional received signal $\mathbf{y}_{0}$ at the tagged SAP 
located at the origin is given by

$$
\begin{aligned}
\mathbf{y}_{0}= & \sqrt{Q_{\mathrm{u}}} r_{0}^{-\frac{\alpha}{2}} \mathbf{g}_{0,0} u_{0}+\sum_{\mathbf{x}_{i} \in \Phi_{\mathrm{s}}^{\mathrm{D}}} \sqrt{P_{\mathrm{s}}}\left\|\mathbf{x}_{0, i}\right\|^{-\frac{\alpha}{2}} \mathcal{H}_{0, i}^{\dagger} \mathbf{W}_{i} \mathbf{x}_{i} \\
& +\sum_{\mathbf{z}_{j} \in \Phi_{\mathrm{u}}^{\mathrm{t}}} \sqrt{Q_{\mathrm{u}}}\left\|\mathbf{z}_{0, j}\right\|^{-\frac{\alpha}{2}} \mathbf{g}_{0, j} u_{j}+\boldsymbol{n}_{\mathbf{0}}
\end{aligned}
$$

where $u_{0}$ and $u_{j}$ are the normalized transmit signal data symbol from the UL MUs located at $\mathbf{z}_{0}$ and $\mathbf{z}_{j}$, respectively, and $\mathbf{x}_{i} \in \mathbb{C}^{M \times M}$ represents the normalized signal vectors of active DL SAPs located at $\mathbf{x}_{i}$. The additive Gaussian noise is given by $\boldsymbol{n}_{\mathbf{0}} \sim \mathcal{C N}\left(0_{M \times 1}, \sigma^{2} \mathbf{I}_{M}\right)$. The channel vector from the typical UL MU located at $\mathbf{z}_{0}$ to the typical SAP is denoted by $\mathbf{g}_{0,0} \in \mathbb{C}^{M \times 1}$ and that from the interfering MU located at $\mathbf{z}_{j}$ is denoted by $\mathbf{g}_{0, j} \in \mathbb{C}^{M \times 1}$. For the interference from DL active SAPs located at $\mathbf{x}_{i}$, the channel vector to the typical $\mathrm{SAP}$ in UL mode is given by $\mathcal{H}_{0, i}^{\dagger} \in \mathbb{C}^{M \times M}$. The SINR at the typical SAP is

$$
\operatorname{SINR}_{\mathrm{U}}=\frac{Q_{\mathrm{u}} r_{0}^{-\alpha}\left\|\mathbf{v}_{0}^{\dagger} \mathbf{g}_{0,0}\right\|^{2}}{I_{\mathrm{D} \rightarrow \mathrm{U}}+I_{\mathrm{U} \rightarrow \mathrm{U}}+\left|\mathbf{v}_{0}^{\dagger} \boldsymbol{n}_{\mathbf{0}}\right|^{2}},
$$

where the aggregate interference $I_{\mathrm{D} \rightarrow \mathrm{U}}$ and $I_{\mathrm{U} \rightarrow \mathrm{U}}$, and the noise power are respectively given by

$$
\begin{aligned}
& I_{\mathrm{D} \rightarrow \mathrm{U}}=\sum_{\mathbf{x}_{i} \in \Phi_{\mathrm{s}}^{\mathrm{D}}} P_{s}\left\|\mathbf{x}_{0, i}\right\|^{-\alpha}\left|\mathbf{v}_{0}^{\dagger} \mathcal{H}_{0, i}^{\dagger} \mathbf{W}_{i}\right|^{2}, \\
& I_{\mathrm{U} \rightarrow \mathrm{U}}=\sum_{\mathbf{z}_{j} \in \Phi_{\mathrm{u}}^{\mathrm{t}}} Q_{\mathrm{u}}\left\|\mathbf{z}_{0, j}\right\|^{-\alpha}\left|\mathbf{v}_{0}^{\dagger} \mathbf{g}_{0, j}\right|^{2}, \quad\left|\mathbf{v}_{0}^{\dagger} \boldsymbol{n}_{\mathbf{0}}\right|^{2}=\sigma^{2} .
\end{aligned}
$$

For the UL active SAPs, the average number of served MUs is $N_{\mathrm{a}} \leq N$ which is a function of $q_{\mathrm{D}}$ and $\Lambda_{\mathrm{u}}$. We consider the ZF receiver, where a unit norm receive filter $\mathbf{v}_{0} \in \mathbb{C}^{M \times 1}$ is chosen orthogonal to the channel vectors of other $N_{\mathrm{a}}-1$ interferer associated with the same tagged SAP, i.e., $\left|\mathbf{v}_{0}^{\dagger} \mathbf{g}_{0, j}\right|^{2}=0$ for $j=1, \ldots, N_{\mathrm{a}}-1 .^{7}$ As is derived in [42], we have $h_{0,0}^{\mathrm{U}}=\|$ $\mathbf{v}_{0}^{\dagger} \mathbf{g}_{0,0} \|^{2} \sim \Gamma\left(M-N_{a}+1,1\right), g_{\mathrm{MU}}^{\mathrm{U}}=\left|\mathbf{v}_{0}^{\dagger} \mathbf{g}_{0, j}\right|^{2} \sim \operatorname{Exp}(1)$, and $g_{\mathbf{x}_{i}, \mathrm{SAP}}^{\mathrm{U}}=\left|\mathbf{v}_{0}^{\dagger} \mathcal{H}_{0, i}^{\dagger} \mathbf{W}_{i}\right|^{2} \sim \Gamma(N, 1)$.

Lemma 2. Given that the typical link length is $r_{0}$, the Laplace transform of interference $I_{\mathrm{D} \rightarrow \mathrm{D}}, I_{\mathrm{D} \rightarrow \mathrm{U}}, I_{\mathrm{U} \rightarrow \mathrm{U}}$, and $I_{\mathrm{U} \rightarrow \mathrm{D}}$ are, respectively, given by

$$
\begin{aligned}
\mathcal{L}_{I_{\mathrm{D} \rightarrow \mathrm{D}}}(s) & =\mathcal{L}_{I_{\mathrm{D} \rightarrow \mathrm{U}}}(s) \\
& \simeq \exp \left(-\pi q_{\mathrm{D}} \lambda_{\mathrm{s}}\left(1-p_{\mathrm{s}, \text { void }}\right)\left(s P_{\mathrm{s}}\right)^{\frac{2}{\alpha}} \mathcal{C}(\alpha, N)\right)
\end{aligned}
$$

$$
\begin{aligned}
\mathcal{L}_{I_{\mathrm{U} \rightarrow \mathrm{U}}}(s) & =\mathcal{L}_{I_{\mathrm{U} \rightarrow \mathrm{D}}}(s) \\
& \simeq \exp \left(-\pi\left(1-q_{\mathrm{D}}\right) N_{\mathrm{a}} \lambda_{\mathrm{S}}\left(s Q_{\mathrm{u}}\right)^{\frac{2}{\alpha}} \delta(\alpha)\right),
\end{aligned}
$$

where

$\mathcal{C}(\alpha, N)=\frac{2}{\alpha} \sum_{k=1}^{N}\left(\begin{array}{l}N \\ k\end{array}\right) \cdot B\left(N-k+\frac{2}{\alpha}, k-\frac{2}{\alpha}\right), \delta(\alpha) \triangleq \frac{2 \pi / \alpha}{\sin (2 \pi / \alpha)}$,

$N_{a}=\left\lceil N\left(1-p_{\mathrm{u}, \text { void }}\right)\right\rceil$, and $B(a, b)=\int_{0}^{1} u^{a-1}(1-u)^{b-1} d u$ is the Beta function.

\footnotetext{
${ }^{7}$ It is worth noting that when $N_{\mathrm{a}}=1$, the $\mathrm{ZF}$ receiver becomes the maximal ratio combining (MRC) technique.
}

Proof: See Appendix B.

Theorem 1. The coverage probability of a typical MU in DL and UL mode is, respectively, given by

$\mathbb{P}_{\mathrm{D}}=\int_{0}^{R_{\mathrm{cl}}} \sum_{i=0}^{M-N} \frac{(-s)^{i}}{i !} \mathcal{L}_{I_{I N}}(s) \sum \frac{i !}{j_{1} ! j_{2} ! \ldots j_{i} !} \prod_{l=1}^{i}\left(\frac{g^{l}(s)}{l !}\right)^{j_{l}} \frac{2 r_{0}}{R_{\mathrm{cl}}^{2}} \mathrm{~d} r_{0}$

$\mathbb{P}_{\mathrm{U}}=\int_{0}^{R_{\mathrm{cl}}} \sum_{i=0}^{M-N_{a}} \frac{(-v)^{i}}{i !} \mathcal{L}_{I_{I N}}(v) \sum \frac{i !}{j_{1} ! j_{2} ! \ldots j_{i} !} \prod_{l=1}^{i}\left(\frac{g^{l}(v)}{l !}\right)^{j_{l}} \frac{2 r_{0}}{R_{\mathrm{cl}}^{2}} \mathrm{~d} r_{0}$,

where $s=\frac{\gamma_{\mathrm{D}} r_{0}^{\alpha}}{P_{\mathrm{s}}}, v=\frac{\gamma_{\mathrm{U}} r_{0}^{\alpha}}{Q_{\mathrm{u}}}, N_{\mathrm{a}}=\left\lceil N\left(1-p_{\mathrm{u}, \text { void }}\right)\right\rceil$, and

$$
\begin{gathered}
\mathcal{L}_{I_{I N}}(x)=\exp \left(-x \sigma^{2}-\pi q_{\mathrm{D}} \lambda_{\mathrm{s}}\left(1-p_{\mathrm{s}, \text { void }}\right)\left(x P_{\mathrm{s}}\right)^{\frac{2}{\alpha}}\right. \\
\left.\cdot \mathcal{C}(\alpha, N)-\pi\left(1-q_{\mathrm{D}}\right) N_{\mathrm{a}} \lambda_{\mathrm{s}}\left(x Q_{\mathrm{u}}\right)^{\frac{2}{\alpha}} \delta(\alpha)\right), \\
g^{l}(x)=-\sigma^{2} 1_{(l=1)}-\prod_{n=0}^{l-1}\left(\frac{2}{\alpha}-n\right) x^{\frac{2}{\alpha}-l}\left[\pi q_{\mathrm{D}} \lambda_{\mathrm{s}}\left(1-p_{\mathrm{s}, \text { void }}\right)\right. \\
\left.\cdot P_{\mathrm{s}}^{\frac{2}{\alpha}} \mathcal{C}(\alpha, N)-\pi\left(1-q_{\mathrm{D}}\right) N_{\mathrm{a}} \lambda_{\mathrm{s}} Q_{\mathrm{u}}^{\frac{2}{\alpha}} \delta(\alpha)\right] .
\end{gathered}
$$

Proof: See Appendix C.

With $\mathbb{P}_{\mathrm{D}}$ and $\mathbb{P}_{\mathrm{U}}$ derived in Theorem 1 , and combining with (12), we derive the optimal UL/DL configuration $q_{\mathrm{D}}^{\star}$ that minimizes the service time in Theorem 2.

Theorem 2. The UL/DL configuration that minimizes the service time of the typical cell is the fixed solution of the following function

$$
\varphi\left(q_{\mathrm{D}}\right) \simeq \begin{cases}\frac{A\left(\mu_{\mathrm{u}, \min }-\Lambda_{\mathrm{u}}+\kappa_{\mathrm{u}}\right)}{\mu_{\mathrm{s}, \min }-\Lambda_{\mathrm{s}}+\kappa_{\mathrm{s}}+A\left(\mu_{\mathrm{u}, \min }-\Lambda_{\mathrm{u}}+\kappa_{\mathrm{u}}\right)}, & \kappa_{\mathrm{s}}=A \kappa_{\mathrm{u}}, \\ \frac{\sqrt{B}-C}{2\left(\kappa_{\mathrm{s}}-A \kappa_{\mathrm{u}}\right)}, & \kappa_{\mathrm{s}} \neq A \kappa_{\mathrm{u}},\end{cases}
$$

where

$$
\begin{aligned}
A \triangleq & \frac{\Lambda_{\mathrm{s}} \mathrm{R}_{\mathrm{U}} \mathbb{P}_{\mathrm{U}}}{\Lambda_{\mathrm{u}} \mathrm{R}_{\mathrm{D}} \mathbb{P}_{\mathrm{D}}} \\
B \triangleq & 4 A\left(\kappa_{\mathrm{s}}-A \kappa_{\mathrm{u}}\right)\left(\mu_{\mathrm{u}, \min }+\kappa_{\mathrm{u}}-\Lambda_{\mathrm{u}}\right) \\
& +\left(\mu_{\mathrm{s}, \min }-\Lambda_{\mathrm{s}}+A\left(\mu_{\mathrm{u}, \min }+2 \kappa_{\mathrm{u}}-\Lambda_{\mathrm{u}}\right)\right)^{2}, \\
C \triangleq & \mu_{\mathrm{s}, \min }-\Lambda_{\mathrm{s}}+A\left(\mu_{\mathrm{u}, \min }+2 \kappa_{\mathrm{u}}-\Lambda_{\mathrm{u}}\right) .
\end{aligned}
$$

Namely, if the optimal UL/DL configuration is $q_{\mathrm{D}}^{\star}$, we have $\varphi\left(q_{\mathrm{D}}^{\star}\right)=q_{\mathrm{D}}^{\star}$.

Proof: According to (11), we have

$$
\begin{aligned}
& \Xi \\
& =\mathbb{E}\left[\frac{B_{\mathrm{s}} \varsigma / \tau_{\mathrm{sf}} \mathrm{R}_{\mathrm{D}} 1_{\left\{\mathrm{SINR}_{\mathrm{D}}>\gamma_{\mathrm{D}}\right\}}}{B_{\mathrm{u} \varsigma} \varsigma \tau_{\mathrm{sf}} \mathrm{R}_{\mathrm{U}} 1_{\left\{\mathrm{SINR}_{\mathrm{U}}>\gamma_{\mathrm{U}}\right\}}}\right] \\
& =\mathbb{E}\left[B_{\mathrm{s}}\right] \cdot \mathbb{E}\left[\frac{1}{B_{\mathrm{u}}}\right] \cdot \frac{\mathrm{R}_{\mathrm{U}}}{\mathrm{R}_{\mathrm{D}}} \cdot \mathbb{E}\left[\frac{1_{\left\{\mathrm{SINR}_{\mathrm{U}}>\gamma_{\mathrm{U}}\right\}}}{1_{\left\{\mathrm{SINR}_{\mathrm{D}}>\gamma_{\mathrm{D}}\right\}}}\right] \\
& \stackrel{(a)}{\geq} \frac{\Lambda_{\mathrm{s}} \mathrm{R}_{\mathrm{U}}\left(\mu_{\mathrm{u}, \min }+\kappa_{\mathrm{u}}\left(1-q_{\mathrm{D}}\right)-\Lambda_{\mathrm{u}}\right)}{\Lambda_{\mathrm{u}} \mathrm{R}_{\mathrm{D}}\left(\mu_{\mathrm{s}, \min }+\kappa_{\mathrm{S}} q_{\mathrm{D}}-\Lambda_{\mathrm{s}}\right)} \cdot \mathbb{E}\left[\frac{1_{\left\{\mathrm{SINR}_{\mathrm{U}}>\gamma_{\mathrm{U}}\right\}}}{1_{\left\{\mathrm{SINR}_{\mathrm{D}}>\gamma_{\mathrm{D}}\right\}}}\right] \\
& \stackrel{(b)}{\simeq} \frac{\Lambda_{\mathrm{s}} \mathrm{R}_{\mathrm{U}} \mathbb{P}_{\mathrm{U}}\left(\mu_{\mathrm{u}, \min }+\kappa_{\mathrm{u}}\left(1-q_{\mathrm{D}}\right)-\Lambda_{\mathrm{u}}\right)}{\Lambda_{\mathrm{u}} \mathrm{R}_{\mathrm{D}} \mathbb{P}_{\mathrm{D}}\left(\mu_{\mathrm{s}, \min }+\kappa_{\mathrm{S}} q_{\mathrm{D}}-\Lambda_{\mathrm{S}}\right)} .
\end{aligned}
$$

where (a) follows by applying Jensen's inequality to the convex function $\frac{1}{x}, x>0$, and (b) follows by ignoring the dependence between $\operatorname{SINR}_{\mathrm{U}}$ and $\mathrm{SINR}_{\mathrm{D}}$, and using the 
Jensen's inequality. Letting $A \triangleq \frac{\Lambda_{\mathrm{s}} R_{\mathrm{U}} \mathbb{P}_{U}}{\Lambda_{\mathrm{u}} \mathrm{R}_{\mathrm{D}} \mathbb{P}_{\mathrm{D}}}$ and combining (12) with (30), we have

$$
\begin{aligned}
& q_{\mathrm{D}}^{2}\left(\kappa_{\mathrm{s}}-A \kappa_{\mathrm{u}}\right)+q_{\mathrm{D}}\left(\mu_{\mathrm{s}, \min }-\Lambda_{\mathrm{s}}\right. \\
& \left.+A\left(\mu_{\mathrm{u}, \min }+2 \kappa_{\mathrm{u}}-\Lambda_{\mathrm{u}}\right)\right)=A\left(\mu_{\mathrm{u}, \min }+\kappa_{\mathrm{u}}-\Lambda_{\mathrm{u}}\right) .
\end{aligned}
$$

By solving the above quadratic equation with regard to $q_{\mathrm{D}}$, we notice that the solution is a function of $A$, which is a function of $\mathbb{P}_{\mathrm{U}}$ and $\mathbb{P}_{\mathrm{D}}$ and thus of $q_{\mathrm{D}}$. We can therefore write the solution of the quadratic equation as $\varphi\left(q_{\mathrm{D}}\right)$ and we derive (29) for the cases $\kappa_{\mathrm{s}}=A \kappa_{\mathrm{u}}$ and $\kappa_{\mathrm{s}} \neq A \kappa_{\mathrm{u}}$. We find that $q_{\mathrm{D}}^{\star}$ is the fixed point of $\varphi\left(q_{\mathrm{D}}\right)$, i.e., $\varphi\left(q_{\mathrm{D}}^{\star}\right)=q_{\mathrm{D}}^{\star}$.

Remark 4. In Theorem 2, it is worth noting that in the derivation of $q_{\mathrm{D}}^{\star}$, we have applied the Jensen's inequality and ignored the dependence between $\operatorname{SINR}_{\mathrm{U}}$ and $\mathrm{SINR}_{\mathrm{D}}$. Therefore, $q_{\mathrm{D}}^{\star}$ is not an exact optimal solution. However, from the simulation results given in Section V, we observe that the approximation has little effect on the accuracy, and $q_{\mathrm{D}}^{\star}$ is very close to the optimal solution.

Remark 5. To prove the coexistence and the uniqueness of the fixed point, we define an auxiliary function $\psi\left(q_{\mathrm{D}}\right)=\varphi\left(q_{\mathrm{D}}\right)-$ $q_{\mathrm{D}}, q_{\mathrm{D}} \in[0,1]$. To guarantee the stability of the queues of the tagged SAP and typical MU, we have $\Lambda_{\mathrm{s}}<\mu_{\mathrm{s}, \min }+\kappa_{\mathrm{s}} q_{\mathrm{D}} \leq$ $\mu_{\mathrm{s}, \min }+\kappa_{\mathrm{s}}$, and $\Lambda_{\mathrm{u}}<\mu_{\mathrm{u}, \min }+\kappa_{\mathrm{u}}\left(1-q_{\mathrm{D}}\right) \leq \mu_{\mathrm{u}, \min }+\kappa_{\mathrm{u}}$. Since $\mathbb{P}_{\mathrm{D}}, \mathbb{P}_{\mathrm{U}} \in(0,1), \Lambda_{\mathrm{s}}, \Lambda_{\mathrm{u}}, \gamma_{\mathrm{D}}$ and $\gamma_{\mathrm{U}}$ are positive parameters, we have $A \in(0, \infty)$. If $\kappa_{\mathrm{s}}=A \kappa_{\mathrm{u}}$, we have $\varphi\left(q_{\mathrm{D}}\right) \in(0,1)$, and thus, $\psi(0)>0$ and $\psi(1)<0$. Similarly, if $\kappa_{\mathrm{s}} \neq A \kappa_{\mathrm{u}}$, we derive that $\psi(0)>0$ and $\psi(1)<0$ based on the stability condition of the queues. Since $\psi\left(q_{\mathrm{D}}\right)$ is continuous within the region $[0,1]$, we derive that there exists $q_{\mathrm{D}}^{\star} \in(0,1)$ such that $\psi\left(q_{\mathrm{D}}^{\star}\right)=0$, i.e., $\varphi\left(q_{\mathrm{D}}^{\star}\right)=q_{\mathrm{D}}^{\star}$, which proves the existence of the fixed point. Due to the complexity of the function, it is difficult to give a formal proof for the uniqueness of $q_{\mathrm{D}}^{\star}$. By resorting to a numerical analysis, we observed the convexity of $\psi\left(q_{\mathrm{D}}\right)$ with all possible parameters, and found that there exists only one $q_{\mathrm{D}}^{\star}$ with $\psi\left(q_{\mathrm{D}}^{\star}\right)=0$.

\section{B. Energy efficiency}

We define the EE as the ratio of traffic load to the total energy consumption, measured in bits/Joule/Hz. For the power consumption, we only account for the energy drawn from the power grid and consider the harvested energy without additional cost. We assume that the sleeping mode is supported by the power grid. Define the overall energy consumption of the tagged SAP with strategy $m$ and the typical MU as $E_{\mathrm{Tot}, \mathrm{s}}^{(m)}$ and $E_{\mathrm{Tot}, \mathrm{u}}$, respectively. Referring to (10), the energy consumption of the tagged SAP with Strategy 1 and Strategy 2 is, respectively, given by

$$
\begin{aligned}
E_{\mathrm{Tot}, \mathrm{S}}^{(1)}= & \tilde{P}_{\mathrm{tr}, \mathrm{N}}\left(1-p_{\mathrm{D}, 1}^{\mathrm{avl}}\right) q_{\mathrm{D}} \tau_{\mathrm{t}}^{\mathrm{D}}+\tilde{P}_{\mathrm{rec}, \mathrm{N}}\left(1-p_{\mathrm{U}, 1}^{\mathrm{avl}}\right)\left(1-q_{\mathrm{D}}\right) \tau_{\mathrm{t}}^{\mathrm{U}} \\
& +P_{\text {sleep }} q_{\mathrm{D}}\left(\tau_{\mathrm{t}}-\tau_{\mathrm{t}}^{\mathrm{D}}\right)+P_{\text {sleep }}\left(1-q_{\mathrm{D}}\right)\left(\tau_{\mathrm{t}}-\tau_{\mathrm{t}}^{\mathrm{U}}\right), \quad(31) \\
E_{\mathrm{Tot}, \mathrm{S}}^{(2)}= & \sum_{i=0}^{N-1} \tilde{P}_{\mathrm{tr}, \mathrm{N}-i} p_{\mathrm{D}, 2, i}^{\mathrm{avl}} q_{\mathrm{D}} \tau_{\mathrm{t}}^{\mathrm{D}}+\sum_{i=0}^{N-1} \tilde{P}_{\mathrm{rec}, \mathrm{N}-i} p_{\mathrm{U}, 2, i}^{\mathrm{avl}}\left(1-q_{\mathrm{D}}\right) \tau_{\mathrm{t}}^{\mathrm{U}} \\
& +P_{\text {sleep }} q_{\mathrm{D}}\left(\tau_{\mathrm{t}}-\tau_{\mathrm{t}}^{\mathrm{D}}\right)+P_{\text {sleep }}\left(1-q_{\mathrm{D}}\right)\left(\tau_{\mathrm{t}}-\tau_{\mathrm{t}}^{\mathrm{U}}\right),
\end{aligned}
$$

where in (32) the probabilities

$$
\begin{aligned}
& p_{\mathrm{D}, 2, i}^{\mathrm{avl}}=\left\{\begin{array}{ll}
1-p_{\mathrm{D}, 2}^{\mathrm{avl}}, & i=0 \\
\nu \pi_{i \omega-1}+(1-\nu) \pi_{i \omega}, & i \leq N-1
\end{array},\right. \\
& p_{\mathrm{U}, 2, i}^{\mathrm{avl}}= \begin{cases}1-p_{\mathrm{U}, 2}^{\mathrm{avl}}, & i=0 \\
\nu \pi_{i}+(1-\nu) \pi_{i} & i \leq N-1\end{cases}
\end{aligned}
$$

respectively, represent the probability that the rechargeable battery can serve $i$ DL or UL data streams, respectively. In (31) and (32), the first two terms consider the energy consumption of an active SAP during DL and UL subframes. ${ }^{8}$ The last two terms consider the energy consumption when the SAP is in sleeping mode. Since the energy consumption of the typical MU is independent of the battery utilization strategy, we derive the energy consumption of the typical MU as

$$
\begin{aligned}
E_{\mathrm{Tot}, \mathrm{u}}= & Q_{\mathrm{rec}} q_{\mathrm{D}} \tau_{\mathrm{t}}^{\mathrm{D}}+Q_{\mathrm{tr}}\left(1-q_{\mathrm{D}}\right) \tau_{\mathrm{t}}^{\mathrm{U}}+Q_{\text {sleep }} \\
& \cdot\left(q_{\mathrm{D}}\left(\tau_{\mathrm{t}}-\tau_{\mathrm{t}}^{\mathrm{D}}\right)+\left(1-q_{\mathrm{D}}\right)\left(\tau_{\mathrm{t}}-\tau_{\mathrm{t}}^{\mathrm{U}}\right)\right) .
\end{aligned}
$$

To show the benefit from traffic adaptation, we define the energy efficiency of the typical small cell as

$$
\mathcal{E E}_{\mathrm{cell}}^{(m)}=\frac{\mathbb{E}\left[B_{\mathrm{s}}\right] \varsigma N+\mathbb{E}\left[B_{\mathrm{u}}\right] \varsigma N_{\mathrm{a}}}{E_{\mathrm{Tot}, \mathrm{s}}^{(m)}+E_{\mathrm{Tot}, \mathrm{u}} N_{\mathrm{a}}}
$$

where the numerator comprises both DL and UL traffic in the small cell, and the denominator represents the total energy consumption of the SAP and active MUs within the cell. We refer to $\mathcal{E E}_{\text {cell }}^{(m)}$ as the network EE. Furthermore, we define the EE of the typical MU and the tagged SAP with strategy $m$ as

$$
\mathcal{E}_{\mathrm{s}}^{(m)}=\frac{\mathbb{E}\left[B_{\mathrm{s}}\right] \varsigma N}{E_{\mathrm{Tot}, \mathrm{s}}^{(m)}}, \quad \mathcal{E} \mathcal{E}_{\mathrm{u}}=\frac{\mathbb{E}\left[B_{\mathrm{u}}\right] \varsigma}{E_{\mathrm{Tot}, \mathrm{u}}} .
$$

\section{Numerical Results}

In this section, we verify first the theoretical model and the approximations therein by means of simulations, and provide then key design insights for energy harvesting multi-antenna small cell networks with dynamic TDD. In this work, the approximations are due to (i) modeling the active MUs in UL mode by a PPP with the same intensity as the original cluster point process, (ii) ignoring the correlation between different subframes during the transmission of the UL and DL traffic, (iii) modeling the intensity of active SAPs in DL mode and active MUs in UL mode by utilizing the void probability of the queue as an independent thinning factor, and (iv) modeling the number of active MUs associated with each UL SAP with an integer.

Before explaining the results, we briefly summarize the simulation procedure as follows: (i) Choose a large spatial window and simulate the locations of each SAP and its associated $N$ MUs as realizations of PPP and the NewmanScott cluster point process of the given intensities. For each realization, we focus on a typical link consisting of a tagged SAP located at the origin and a typical MU randomly selected from the associated MU set. (ii) Generate the queue length for all the SAPs and MUs according to the geometric distribution

\footnotetext{
${ }^{8}$ To simplify the analysis, in the second term, we consider the worst case where all the $N$ associated UL MUs are active.
} 


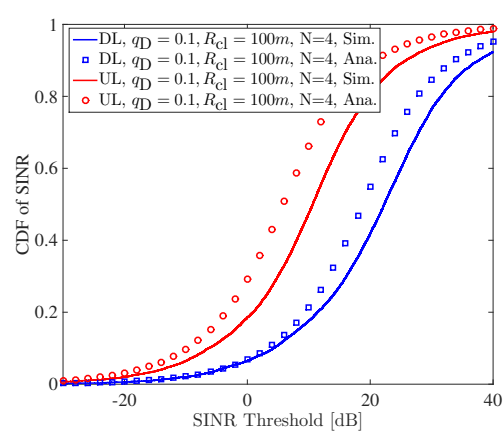

(a)

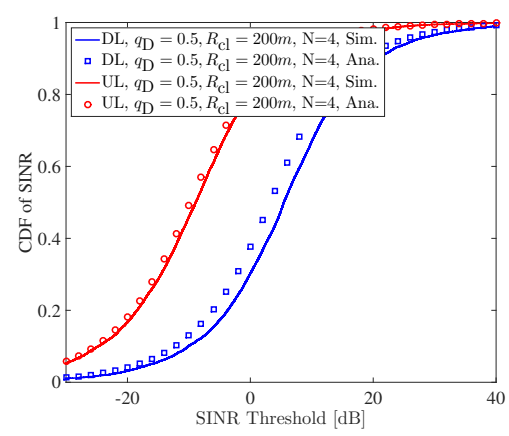

(d)

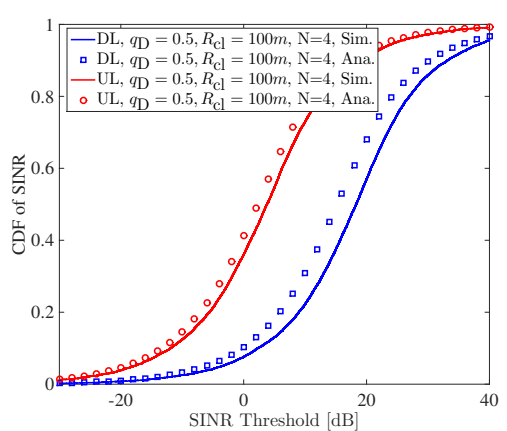

(b)

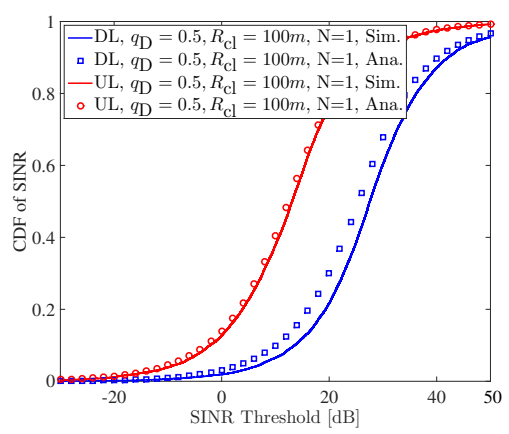

(e)

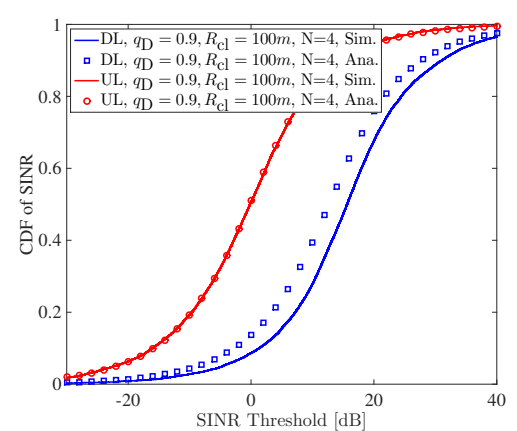

(c)

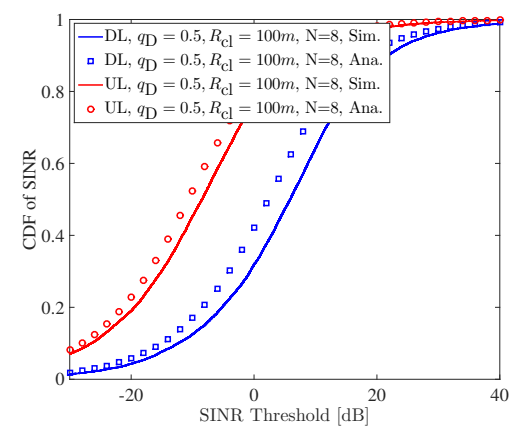

(f)

Figure 3. Comparison of coverage probability from simulations and theoretical analysis as a function of the SINR threshold $\gamma_{\mathrm{D}}\left(\gamma_{\mathrm{U}}\right)$ in the full-buffer case, for $\alpha=4, M=8, \lambda_{\mathrm{s}}=10^{-5} \mathrm{~m}^{-2}, P_{\mathrm{s}}=200 \mathrm{~mW}, Q_{s}=10 \mathrm{~mW}, \sigma^{2}=-121 \mathrm{dBm}$.

of given parameters. If the queue length of a DL SAP or a UL MU is empty, then the SAP or MU enters into sleeping mode. (iii) In each subframe, an SAP (including the tagged SAP) is selected to operate in DL or UL mode with the given probabilities $q_{\mathrm{D}}$ or $1-q_{\mathrm{D}}$, respectively. Generate the fading r.v.'s $h_{0,0}^{\mathrm{D}}$ or $h_{0,0}^{\mathrm{U}}$ for the typical link according to $\Gamma(M-N+1,1)$ or $\Gamma\left(M-N_{\mathrm{a}}+1,1\right)$ when the tagged SAP operates in DL or UL mode. Generate the fading r.v.'s $g_{\mathbf{x}_{i}, \mathrm{SAP}}^{\mathrm{D}}$ and $g_{\mathrm{MU}}^{\mathrm{D}}$ for the remaining active DL SAPs and active transmitting MUs according to $\Gamma(N, 1)$ and $\operatorname{Exp}(1)$, respectively. (iv) In each subframe, we only update the queue length of the DL tagged SAP or the typical UL MU while keeping the queue length of all the remaining DL SAPs and UL MUs unchanged, in accordance with the idea of a stationary distribution. (v) If both queues of the tagged SAP and the typical MU are empty, record the number of total consumed frames and go to (i). Otherwise, if the queue length of the tagged SAP (typical MU) is empty, the tagged SAP (typical MU) enters sleeping mode in the DL (UL) subframes. We choose system parameters that are compatible with TD-LTE, where each radio frame consists of ten subframes with the duration of one subframe being $1 \mathrm{~ms}$, and the system bandwidth is chosen as $W=200$ $\mathrm{kHz}$, approximately corresponding to one resource block in the frequency domain. For the EE calculation, we consider the following default power values [in W]: $P_{\mathrm{s}}=0.2, P_{\mathrm{c}}=6.8$, $P_{0, \mathrm{D}}=6.5, P_{\text {sleep }}=4.3, \eta_{\mathrm{s}}=0.25, \eta_{\mathrm{u}}=0.25, P_{\mathrm{dec}}=0.2$, $P_{\text {mud }}=0.2, P_{0, \mathrm{U}}=0.4, P_{\mathrm{r}}=0.1, Q_{\mathrm{u}}=0.01, Q_{\mathrm{c}}=0.02$, $Q_{0, \mathrm{U}}=0.08, Q_{\mathrm{r}}=0.01, Q_{\mathrm{dec}}=0.01, Q_{0, \mathrm{D}}=0.01$, $Q_{\text {sleep }}=0.01$.

\section{A. Coverage probability}

In Fig. 3, we verify the PPP approximation of active MUs in UL mode in terms of cumulative distribution function (CDF) of the coverage probability in the full-buffer case by varying the SINR threshold for different $q_{\mathrm{D}}, R_{\mathrm{cl}}$, and $N$. By comparing Fig. 3(a), Fig. 3(b) and Fig. 3(c), we observe that a larger $q_{\mathrm{D}}$ leads to a more accurate approximation. This can be understood by the fact that a lower intensity of UL MUs reduces the effect of the PPP approximation. Comparing Fig. 3(b) and Fig. 3(d), we observe that the larger the cluster radius $R_{\mathrm{cl}}$ is, the more accurate the approximation is. As the cluster size increases, the cluster point process approaches to a PPP of the same intensity. By comparing Fig. 3(b), Fig. 3(e) and Fig. 3(f), we derive that a larger number of served MUs $N$ decreases the accuracy of the approximation, which indicates the decreasing quality of the PPP approximation. In this example, the largest gap is achieved by the UL coverage probability in Fig. 3(a), where the order of magnitude of the largest error is less than $20 \%$.

Figure 4 depicts the DL (Fig. 4(a)) and UL (Fig. 4(b)) coverage probabilities for different DL packet arrival rates $\Lambda_{\mathrm{s}}$. We observe a good match between the simulation and numerical analysis for both the lightly and heavily loaded cases, where the order of magnitude of the largest error is less than 5\%. As $q_{\mathrm{D}}$ increases, we observe that the coverage probability with $\Lambda_{\mathrm{s}}=0.1 \Lambda_{\mathrm{u}}$ increases while the coverage probability with $\Lambda_{\mathrm{s}}=9 \Lambda_{\mathrm{u}}$ decreases. This can be understood by the fact that with $\Lambda_{\mathrm{s}}=0.1 \Lambda_{\mathrm{u}}$, the coverage probability is dominated by the strong interference from UL MUs. A larger 


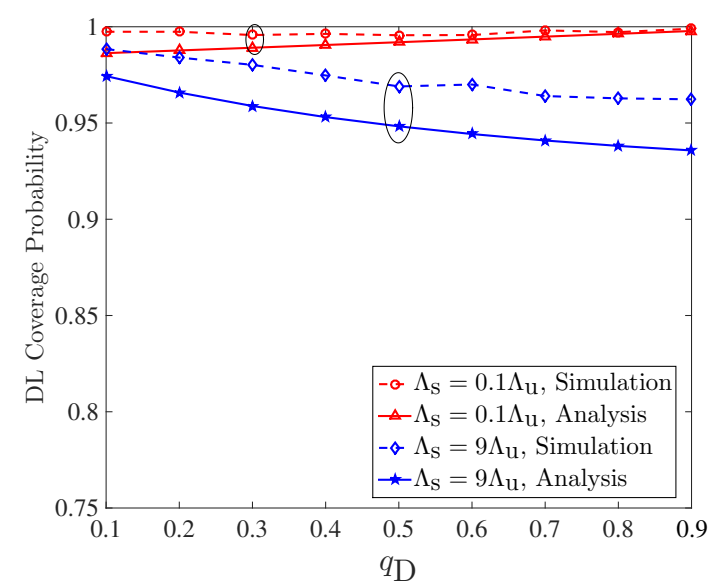

(a)

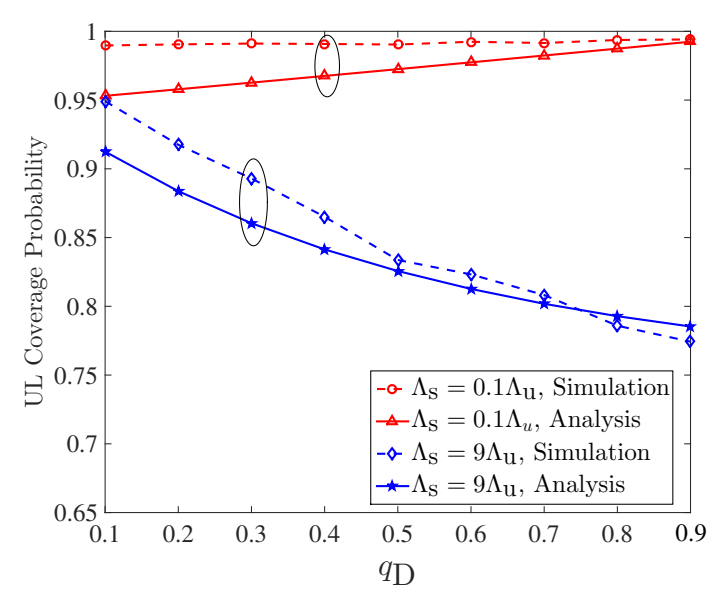

(b)

Figure 4. Comparison of the coverage probability as a function of $q_{\mathrm{D}}$ from simulations and theoretical analysis in the load aware model for different $\Lambda_{\mathrm{S}}$, with $\alpha=4,\left\{\gamma_{\mathrm{D}}, \gamma_{\mathrm{U}}\right\}=\{0,0\}[\mathrm{dB}], R_{\mathrm{cl}}=100 \mathrm{~m}, \lambda_{\mathrm{s}}=10^{-5} \mathrm{~m}^{-2}$, $\Lambda_{\mathrm{u}}=0.05, \mu_{\mathrm{s}, \min }=0.5, \mu_{\mathrm{u}, \min }=0.2, \kappa_{\mathrm{s}}=\kappa_{\mathrm{u}}=0.5$.

$q_{\mathrm{D}}$ decreases the UL interference, resulting in an improvement in the corresponding coverage probability. On the contrary, when $\Lambda_{\mathrm{s}}=9 \Lambda_{\mathrm{u}}$, the DL interference is the limiting factor to the coverage probability. An increasing $q_{\mathrm{D}}$ leads to the growth in DL interference, which reduces the coverage probability.

\section{B. UL/DL configuration vs. traffic adaptation}

In Fig. 5, we present the DL and UL service time (Fig. 5(a)), and the cell service time for different DL packet arrival rates $\Lambda_{\mathrm{S}}$ as a function of $q_{\mathrm{D}}$ where we set $\Lambda_{\mathrm{u}}=0.05$ unchanged. We observe a good match between the simulations and the analytical results. This is mainly due to the fact that we keep the queue length of the remaining DL SAPs and the UL MUs unchanged, which weakens the temporal correlations between different subframes. From Fig. 5(a), we observe that an increasing $\Lambda_{\mathrm{S}}$ increases DL service time while has a little effect on the UL service time. This can be explained that a larger $\Lambda_{\mathrm{s}}$ leads to a heavier DL traffic load, which increases both the expectation of the SAP's queue length and the active DL SAP's intensity. The slight increase of the UL service time

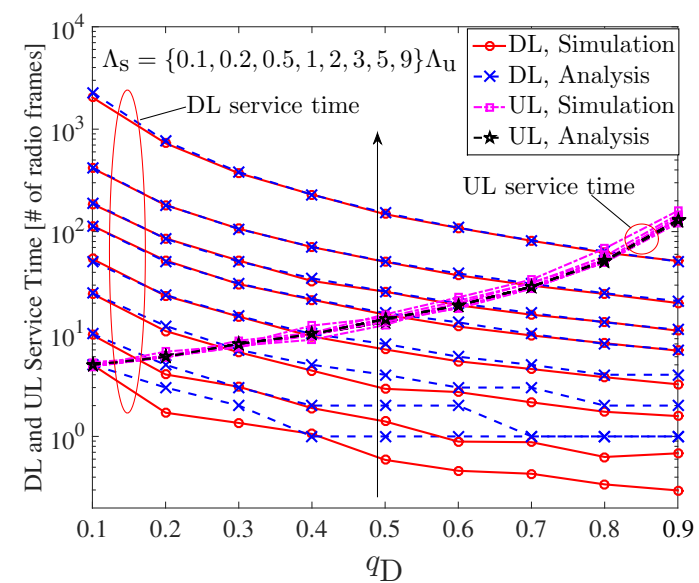

(a)

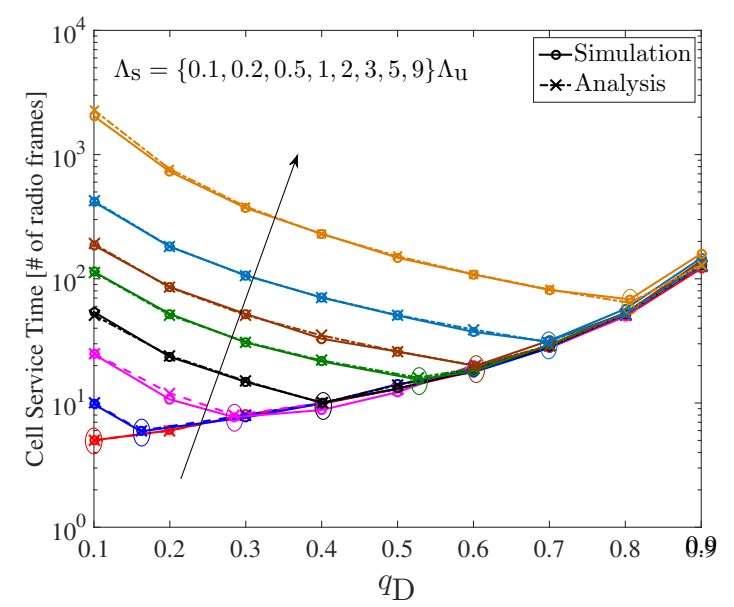

(b)

Figure 5. Service time as a function of $q_{\mathrm{D}}$ from simulations and theoretical analysis with $\alpha=4, \lambda_{\mathrm{s}}=10^{-5} \mathrm{~m}^{-2}, R_{\mathrm{cl}}=100 \mathrm{~m}, \Lambda_{\mathrm{u}}=0.05, \mu_{\mathrm{s}, \min }=$ $0.5, \mu_{\mathrm{u}, \min }=0.2, \kappa_{\mathrm{s}}=\kappa_{\mathrm{u}}=0.5, W=200 \mathrm{kHz}, \varsigma=100 \mathrm{~kb},\left\{\gamma_{\mathrm{D}}, \gamma_{\mathrm{U}}\right\}=$ $\{0,0\}[\mathrm{dB}]$, for (a) DL and UL service time, (b) Cell service time.

is caused by the decreasing UL coverage probability which is caused by the increasing DL interference. We also observe that as $q_{\mathrm{D}}$ increases, the DL service time decreases while the UL service time increases. This is due to the fact that as $q_{\mathrm{D}}$ increases, the DL service rate $\mu_{\mathrm{s}}$ increases, leading to a decrease in the DL service time. Opposite effects hold for UL transmissions. With $\Lambda_{\mathrm{u}}$ unchanged, for each setting of $\Lambda_{\mathrm{s}}$ there exists an optimal UL/DL configuration $q_{\mathrm{D}}^{\star}$ that minimizes the cell service time. It is achieved at the intersection of the two corresponding curves as illustrated in Fig. 5(b). From Fig. 5(b), we observe that the optimal $q_{\mathrm{D}}^{\star}$ shifts to the right as $\Lambda_{\mathrm{s}}$ increases, which can be understood by the fact that giving more time resources to the DL traffic results in an increase of the DL service rate and a corresponding reduction of DL service time.

The optimal $q_{\mathrm{D}}^{\star}$ for each packet arrival rate setting can be derived by solving the fixed point equation (29), which is given in Table II denoted by "Analysis". In practical deployment, the UL/DL configurations can not be set arbitrarily but restricted within a discrete set. In this work, we consider nine values 


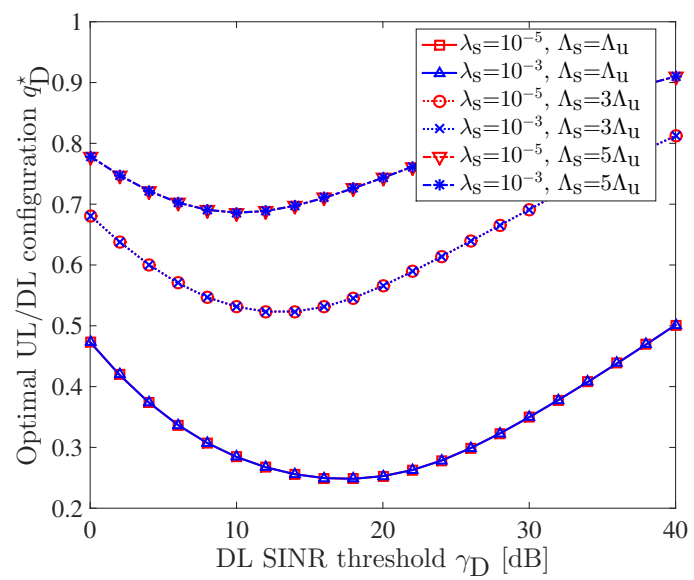

(a)

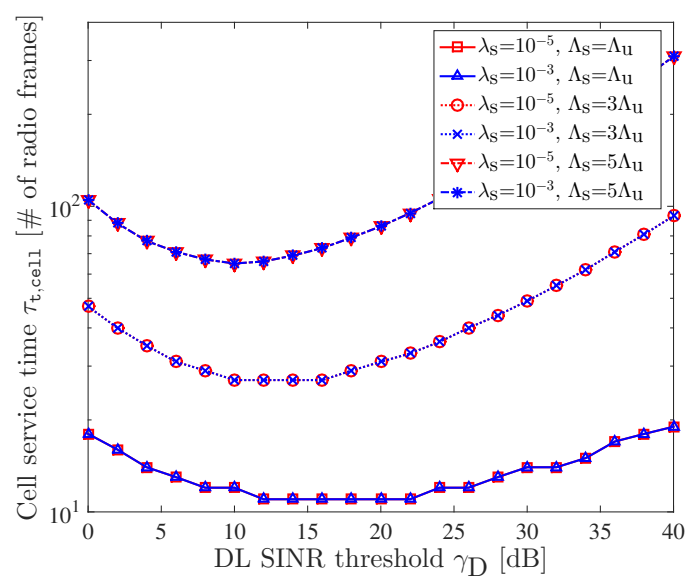

(b)

Figure 6. The optimal UL/DL configuration $q_{\mathrm{D}}^{\star}$ and cell service time $\tau_{\mathrm{t} \text {,cell }}$ as a function of DL SINR threshold $\gamma_{\mathrm{D}}$ for different $\lambda_{\mathrm{s}}$ and $\Lambda_{\mathrm{S}}$ with $\alpha=4$, $\varsigma=100 \mathrm{~kb}, \gamma_{\mathrm{U}}=0 \mathrm{~dB}, \mu_{\mathrm{s}, \min }=\mu_{\mathrm{u}, \min }=0.5, \kappa_{\mathrm{s}}=\kappa_{\mathrm{u}}=0.5$, $W=200 \mathrm{kHz}, \Lambda_{\mathrm{u}}=0.1, R_{\mathrm{cl}}=\frac{1}{\sqrt{\pi \lambda_{\mathrm{s}}}}$ and $\sigma^{2}=0$.

for $q_{\mathrm{D}}$ from 0.1 to 0.9 . By comparing the cell service time achieved at each value of $q_{\mathrm{D}}$, we derive the "Practical Value" of optimal $q_{\mathrm{D}}$ in Table II, which shows a good match to the analytical optimal $q_{\mathrm{D}}^{\star}$.

Figure 6 depicts $q_{\mathrm{D}}^{\star}$ and $\tau_{\mathrm{t} \text {,cell }}$ as a function of $\gamma_{\mathrm{D}}$ for different $\lambda_{\mathrm{s}}$ in the interference-limited regime. An increasing $\Lambda_{\mathrm{S}}$ leads to an increase in both $q_{\mathrm{D}}^{\star}$ and $\tau_{\mathrm{t}, \text { cell }}$, which is caused by the increased asymmetric DL traffic load to the UL traffic load. Keeping the network traffic load unchanged, we observe that both $q_{\mathrm{D}}^{\star}$ and $\tau_{\mathrm{t} \text {,cell }}$ are independent of $\lambda_{\mathrm{s}}$ when $R_{\mathrm{cl}}$ is set according to the average coverage area of each SAP $\pi R_{\mathrm{cl}}^{2}=\lambda_{\mathrm{s}}^{-1}$. This can be understood since $\mathbb{P}_{\mathrm{D}}$ and $\mathbb{P}_{\mathrm{U}}$ remain constant in the interference-limited regime as the change of the received signal power due to the variation in the density of SAPs is balanced by the change in the interference power. Note that the same conclusion is derived within the fullyloaded model in [6], which means that by removing the DL SAPs and UL MUs with empty queues, the network with active DL SAPs and UL MUs can be seen as an equivalent fully-loaded network. From Fig. 6(a), we observe that $q_{\mathrm{D}}^{\star}$ is a

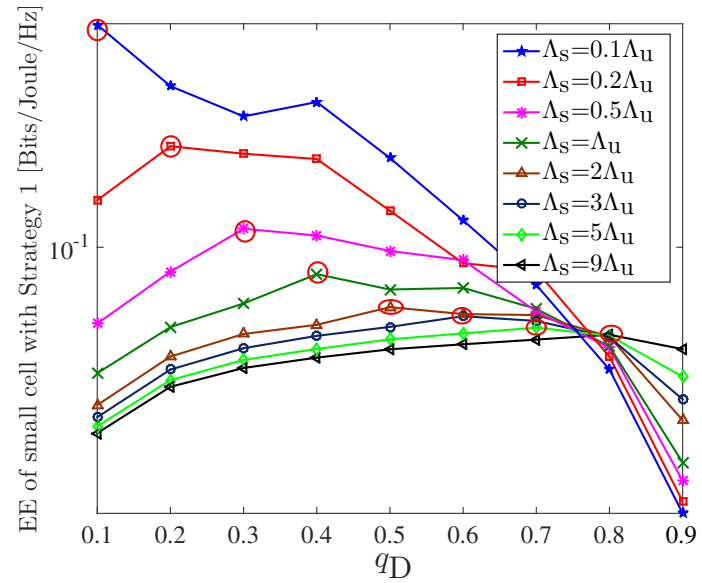

(a)

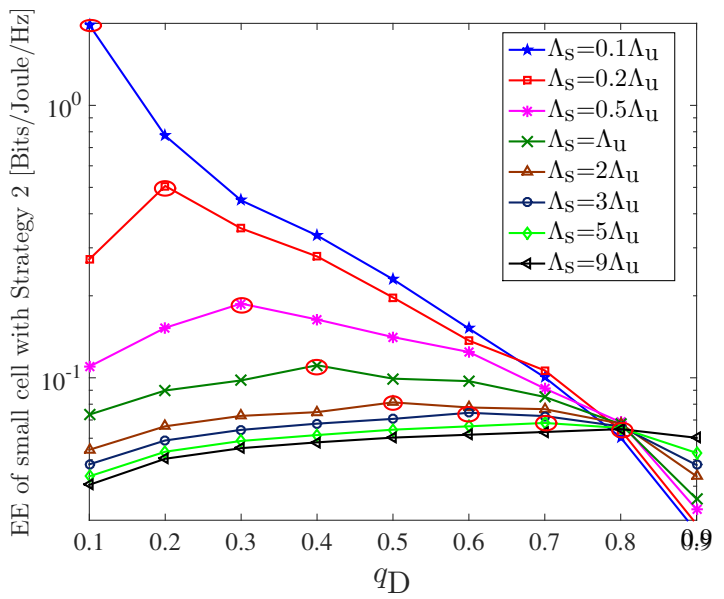

(b)

Figure 7. EE of the typical small cell as a function of $q_{\mathrm{D}}$ with $\alpha=4$, $\varsigma=100 \mathrm{~kb}, \lambda_{\mathrm{s}}=10^{-5} \mathrm{~m}^{-2}, \Lambda_{\mathrm{u}}=0.05, \mu_{\mathrm{s}, \min }=0.5, \mu_{\mathrm{u}, \min }=0.2$, $R_{\mathrm{cl}}=100 \mathrm{~m}, \kappa_{\mathrm{s}}=\kappa_{\mathrm{u}}=0.5, W=200 \mathrm{kHz},\left\{\gamma_{\mathrm{D}}, \gamma_{\mathrm{U}}\right\}=\{0,0\}[\mathrm{dB}],(\mathrm{a})$ for SAP with Strategy 1, (b) for SAP with Strategy 2.

convex function of $\gamma_{\mathrm{D}}$. This can be explained by the tradeoff between the coverage probability $\mathbb{P}_{\mathrm{D}}$ and the target rate $\mathrm{R}_{\mathrm{D}}$, which leads to a nontrivial behavior in the DL outage capacity $R_{D} \mathbb{P}_{D}$. Specifically, an increasing $\gamma_{\mathrm{D}}$ yields a decrease of $\mathbb{P}_{\mathrm{D}}$ and a logarithmic increase of $\mathrm{R}_{\mathrm{D}}$. Figure $6(\mathrm{~b})$ displays $\tau_{\mathrm{t}, \text { cell }}$ as a function of $\gamma_{\mathrm{D}}$ and reveals a similar tendency as $q_{\mathrm{D}}^{\star}$ in Fig. 6(a). The minimal $\tau_{\mathrm{t}, \text { cell }}$ and $q_{\mathrm{D}}^{\star}$ are achieved at the $\gamma_{\mathrm{D}}$ where the DL outage capacity is maximized. Compared with the case $\gamma_{\mathrm{D}}=\gamma_{\mathrm{U}}$, the optimal setting of $\gamma_{\mathrm{D}}$ results in an reduction of as much as $60 \%$ of the service time. These results demonstrate that the proposed analytical framework can be used to derive the optimal SINR threshold that minimizes the service time of the tagged small cell.

\section{Traffic adaptation vs. energy efficiency}

Fig. 7 depicts the EE of the typical small cell as a function of $q_{\mathrm{D}}$ with Strategy 1 (Fig. 7(a)) and with Strategy 2 (Fig. 7(b)), respectively. Compared to the results given in Table II, we observe that the optimal UL/DL configuration $q_{\mathrm{D}}^{\star}$ that minimizes the service time also leads to the optimal EE of 
Table II

THE OPTIMAL UL/DL CONFIGURATION $q_{\mathrm{D}}^{\star}$ WITH $\Lambda_{\mathrm{u}}=0.05$.

\begin{tabular}{|c|c|c|c|c|c|c|c|c|}
\hline$\Lambda_{\mathrm{s}}$ & 0.005 & 0.01 & 0.025 & 0.05 & 0.1 & 0.15 & 0.25 & 0.45 \\
\hline \hline Analysis & 0.0967 & 0.1618 & 0.2849 & 0.4008 & 0.5272 & 0.6026 & 0.6970 & 0.8058 \\
\hline Practical Value & 0.1 & 0.2 & 0.3 & 0.4 & 0.5 & 0.6 & 0.7 & 0.8 \\
\hline
\end{tabular}

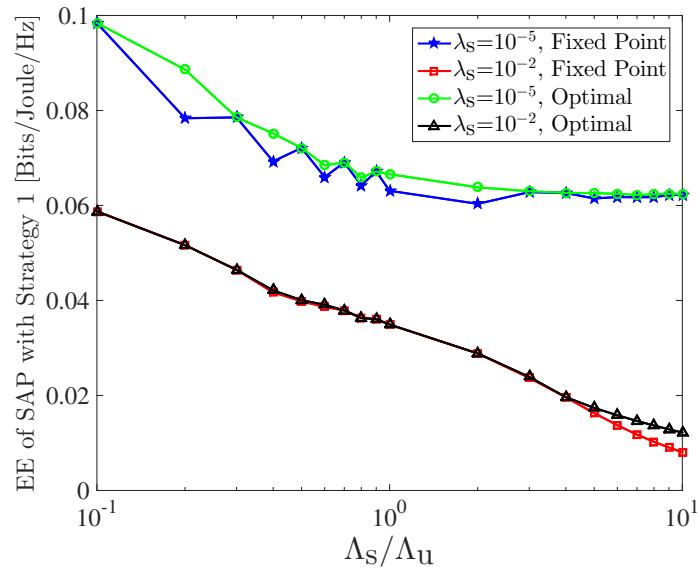

(a)

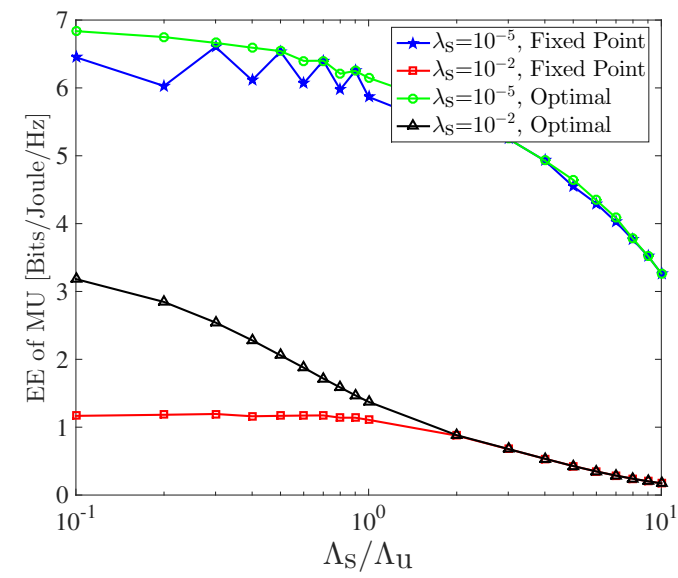

(b)

Figure 8. EE of SAP and MU as a function of $q_{\mathrm{D}}$ with $\alpha=4, \varsigma=100 \mathrm{~kb}$ $\lambda_{\mathrm{s}}=10^{-5} \mathrm{~m}^{-2}, \Lambda_{\mathrm{u}}=0.05, \mu_{\mathrm{s}, \min }=0.5, \mu_{\mathrm{u}, \min }=0.2, R_{\mathrm{cl}}=100 \mathrm{~m}$, $\kappa_{\mathrm{s}}=\kappa_{\mathrm{u}}=0.5, W=200 \mathrm{kHz},\left\{\gamma_{\mathrm{D}}, \gamma_{\mathrm{U}}\right\}=\{0,0\}[\mathrm{dB}]$, (a) for SAP with Strategy 1, (b) for MU.

the typical small cell in each setting of packet arrival rates. ${ }^{9}$ This can be explained by the fact that by conducting traffic adaptation, the tagged SAP can serve the total amount of traffic in the typical small cell with the minimal service time. Therefore, the consumed energy is minimized and the EE is optimized.

In Fig. 8, we separately represent the EE for the tagged SAP and the typical MU achieved at the fixed point and the optimal value derived via a linear search method. We observe that $q_{\mathrm{D}}^{\star}$ does not necessarily lead to the optimal EE of either SAP or MU due to the fact that we focus on the traffic in

${ }^{9}$ Note that the small variance between the optimal point from the fixed point is due to the approximations considered in the analytical results.

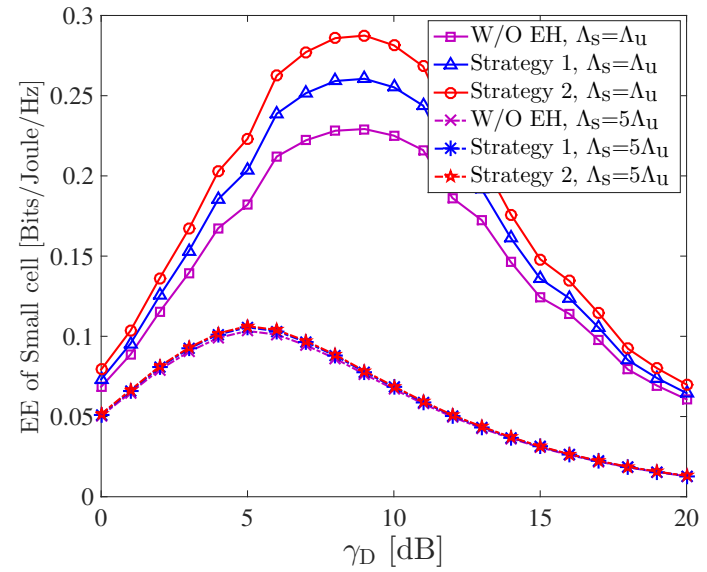

(a)

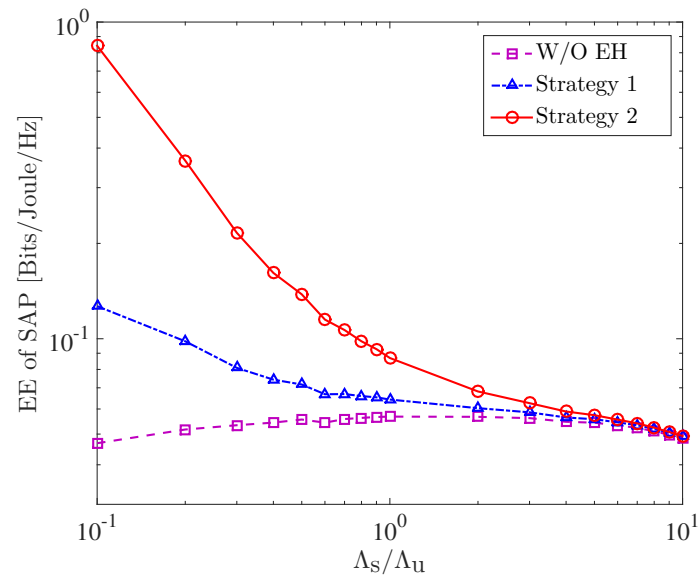

(b)

Figure 9. The optimal network EE and EE of the tagged SAP with $\alpha=4$, $\varsigma=100 \mathrm{~kb}, \gamma_{\mathrm{U}}=0 \mathrm{~dB}, \nu=0.5, \lambda_{\mathrm{s}}=10^{-3}, \mu_{\mathrm{s}, \min }=\mu_{\mathrm{u}, \min }=0.5$, $\kappa_{\mathrm{s}}=\kappa_{\mathrm{u}}=0.5, W=200 \mathrm{kHz}, R_{\mathrm{cl}}=\frac{1}{\sqrt{\pi \lambda_{\mathrm{s}}}}$ and $\sigma^{2}=0$, for (a) $\Lambda_{\mathrm{u}}=0.1$, for (b) $\Lambda_{\mathrm{u}}=0.05$, and $\left\{\gamma_{\mathrm{D}}, \gamma_{\mathrm{U}}\right\}=\{0,0\} \mathrm{dB}$.

both UL and DL rather than either one of them individually. From Fig. 8, we derive that the EE of both SAP and MU decreases with $\Lambda_{\mathrm{s}}$. The decreasing EE of SAP results from the fact that the increasing DL traffic load leads to a reduction of the availability of the battery. The decreasing EE of MU can be understood by considering that increasing DL interference results in a decrease in the UL coverage probability $\mathbb{P}_{U}$, and more energy is needed for successfully transmit each packet. We also observe that an increasing $\lambda_{\mathrm{s}}$ leads to a decreasing EE which can be explained by the reduced coverage probability. 


\section{Effect of energy harvesting on energy efficiency}

To illustrate the benefit from energy harvesting, we present the optimal network EE and the EE of an SAP for different battery utilization strategies in the interference-limited regime in Fig. 9, where the EE without energy harvesting (denoted by "W/O EH") is given as a benchmark. Similar to Fig. 6, the $\mathrm{EE}$ is shown to be independent of $\lambda_{\mathrm{s}}$. Compared to the case without energy harvesting, we observe that equipping the SAP with energy harvesting capabilities can greatly improve both the network EE and the EE of an SAP especially when $\Lambda_{\mathrm{s}}$ is small, and the gain is reduced as $\Lambda_{\mathrm{s}}$ increases. The EE achieved by Strategy 2 is larger than that of Strategy 1, which means that utilizing the battery whenever available improves the EE. From Fig. 9(a), we observe that a proper setting of $\gamma_{\mathrm{D}}$ can significantly improve the network EE and the gain from energy harvesting in the low DL traffic load regime. When $\lambda_{\mathrm{S}}=\lambda_{\mathrm{u}}$, an optimal setting of $\gamma_{\mathrm{D}}$ results in an improvement of $230 \%$ of the EE without energy harvesting. With energy harvesting, the improvement can be further enhanced as much as $25 \%$. In Fig. 9(b), without energy harvesting, we observe that the EE of SAP exhibits a concave behavior of $\gamma_{\mathrm{D}}$. This can be explained by the different effects of $q_{\mathrm{D}}$ on $\mathbb{P}_{\mathrm{D}}$ with different DL traffic loads, which is verified in Fig. 4. Specifically, in the low DL traffic load region (e.g., $\Lambda_{\mathrm{s}} \leq \Lambda_{\mathrm{u}}$ ), $\mathbb{P}_{\mathrm{D}}$ is dominated by the UL interference, and increasing $q_{\mathrm{D}}$ results in a lower UL interference, and correspondingly a larger $\mathbb{P}_{\mathrm{D}}$. Conversely, in the heavy DL traffic load region (e.g., $\Lambda_{\mathrm{s}}>\Lambda_{\mathrm{u}}$ ), opposite effects can be observed. The numerical analysis presented in Fig. 9 shows that the $q_{\mathrm{D}}$ that achieves the optimal EE of an SAP grows with $\Lambda_{\mathrm{s}}$. In the low DL traffic load region, the positive effect of $q_{\mathrm{D}}$ on $\mathbb{P}_{\mathrm{D}}$ is larger than the negative effect of $\Lambda_{\mathrm{S}}$ on $\mathbb{P}_{\mathrm{D}}$, which leads to the rising EE of an SAP. While in the high DL traffic load region, both the augmentation of $q_{\mathrm{D}}$ and $\Lambda_{\mathrm{s}}$ reduces $\mathbb{P}_{\mathrm{D}}$, which leads to the drop of the EE.

\section{Conclusions}

In this work, we studied traffic adaptation in a multi-antenna energy harvesting small cell network, where the SAPs operate dynamic TDD and utilize harvested energy to reduce the use of conventional energy. We proposed a stylized model that allows us to get insight into the main trends in traffic adaptation, and verified the accuracy of the model by extensive simulations. Given the queue length distribution of SAPs and MUs, we first derived the DL and UL coverage probabilities under a given UL/DL configuration and then determined the optimal UL/DL configuration from the perspective of the service time of the typical small cell. By designing several battery utilization strategies, we derived the availability of the battery and quantified the effect of energy harvesting on EE. We observed that energy harvesting can bring more benefit to the EE especially in the low traffic load regime. Furthermore, we shed light on the relationship between traffic adaptation and EE, and showed that the UL/DL configuration that minimizes the service time also leads to an optimal network EE, but does not necessarily yield optimal EE for an SAP or an MU individually. We showed the power of our analytical framework by optimally setting the SINR threshold, which leads to a significant improvement in network EE and reduction in service time. Our analysis quantifies the potential benefits of traffic adaptation and the use of energy harvesting technique in multi-antenna small cell networks with dynamic TDD.

\section{APPENDIX}

\section{A. Derivations of transition probabilities}

For Strategy 1, the transition probability from state $k$ to state $k$ is given by

$p_{k, k}= \begin{cases}1-\nu, & 0 \leq k \leq N-1 \\ (1-\nu)\left(q_{\mathrm{D}}+\left(1-q_{\mathrm{D}}\right) p_{\mathrm{u}, \text { void }}\right), & N \leq k \leq \omega N-1 \\ (1-\nu)\left(q_{\mathrm{D}} p_{\mathrm{s}, \text { void }}+\left(1-q_{\mathrm{D}}\right) p_{\mathrm{u}, \text { void }}\right), & \omega N \leq k \leq K-1 \\ q_{\mathrm{D}} p_{\mathrm{s}, \text { void }}+\left(1-q_{\mathrm{D}}\right) p_{\mathrm{u}, \text { void }}, & k=K\end{cases}$

where the necessary condition leading to $p_{k, k}$ is that no energy arrives at the given SAP when $k<K$. The first three equations in the right hand of (38) respectively consider the cases where the harvested energy can support (i) neither DL nor UL transmissions, (ii) only UL transmissions, (iii) both $\mathrm{UL}$ and DL transmissions. When $k=K$, the battery is fully charged and $p_{k, k}$ is independent of the energy arrival process, and the transition from state $k$ to $k$ is due to that there is no traffic in the typical small cell.

The transition from state $k$ to state $k+1$ is given by

$p_{k, k+1}= \begin{cases}\nu, & 0 \leq k \leq N-2 \\ \nu\left(q_{\mathrm{D}}+\left(1-q_{\mathrm{D}}\right) p_{\mathrm{u}, \text { void }}\right), & N-1 \leq k \leq \omega N-2 \\ \nu\left(q_{\mathrm{D}} p_{\mathrm{s}, \text { void }}+\left(1-q_{\mathrm{D}}\right) p_{\mathrm{u}, \text { void }}\right), & \omega N-1 \leq k \leq K-1\end{cases}$

where the necessary condition for $p_{k, k+1}$ is that there is energy arriving at the SAP in the incoming subframe when $k<K$. The explanations for each equation in the right hand are similar to the statements for $p_{k, k}$ in (38) and thus omitted.

When serving $N$ UL data streams, the state can be transited from $k$ to $k-(N-1)$ or from $k$ to $k-N$ depending on whether energy arrives at the SAP. The corresponding transition probabilities are given by

$$
\begin{gathered}
p_{k, k-(N-1)}=\nu\left(1-q_{\mathrm{D}}\right)\left(1-p_{\mathrm{u}, \mathrm{void}}\right), N-1 \leq k \leq K, \\
p_{k, k-N}=(1-\nu)\left(1-q_{\mathrm{D}}\right)\left(1-p_{\mathrm{u}, \mathrm{void}}\right), N \leq k \leq K,
\end{gathered}
$$

Similarly, when the harvested energy can support $N$ DL data streams, the state can be transited from $k$ to $k-(\omega N-1)$ or from $k$ to $k-\omega N$ based on the energy arrival cases in next subframe. We have

$$
\begin{gathered}
p_{k, k-(\omega N-1)}=\nu q_{\mathrm{D}}\left(1-p_{\mathrm{s}, \text { void }}\right), \omega N-1 \leq k \leq K, \\
p_{k, k-\omega N}=(1-\nu) q_{\mathrm{D}}\left(1-p_{\mathrm{s}, \text { void }}\right), \omega N \leq k \leq K,
\end{gathered}
$$

Different from Strategy 1, in Strategy 2 the rechargeable battery is available to DL and UL transmissions as long as the harvested energy can afford one data stream in the corresponding transmission mode. Since the power consumption for a DL data stream is usually much higher than that for a UL data stream, we consider the case where $\omega>N$ with $N$ is 
the number of served MUs within a small cell. The transition probability from state $k$ to state $k$ is given by

$$
p_{k, k}= \begin{cases}1-\nu+\nu\left(1-q_{\mathrm{D}}\right)\left(1-p_{\mathrm{u}, \text { void }}\right), & k=0 \\ (1-\nu)\left(q_{\mathrm{D}}+\left(1-q_{\mathrm{D}}\right) p_{\mathrm{u}, \text { void }}\right), & 1 \leq k \leq \omega-1 \\ (1-\nu)\left(q_{\mathrm{D}} p_{\mathrm{s}, \text { void }}+\left(1-q_{\mathrm{D}}\right) p_{\mathrm{u}, \text { void }}\right), & \omega \leq k \leq K-1 \\ q_{\mathrm{D}} p_{\mathrm{s}, \text { void }}+\left(1-q_{\mathrm{D}}\right) p_{\mathrm{u}, \text { void }}, & k=K\end{cases}
$$

The transition from state $k$ to state $k+1$ is given by

$$
p_{k, k+1}= \begin{cases}\nu\left(q_{\mathrm{D}}+\left(1-q_{\mathrm{D}}\right) p_{\mathrm{u}, \text { void }}\right), & 0 \leq k \leq \omega-2 \\ \nu\left(q_{\mathrm{D}} p_{\mathrm{s}, \text { void }}+\left(1-q_{\mathrm{D}}\right) p_{\mathrm{u}, \text { void }}\right), & \omega-1 \leq k \leq K-1\end{cases}
$$

where the two equations consider the case when the harvested energy is only available to UL transmissions and available to both UL and DL transmissions. An SAP may use up the harvested energy to serve $k$ or $k+1$ UL data streams when the harvested energy level $k \leq N-1$. The transition probability $p_{k, 0}$ is

$$
p_{k, 0}=\left(1-q_{\mathrm{D}}\right)\left(1-p_{\mathrm{u}, \mathrm{void}}\right), 1 \leq k \leq N-1 .
$$

Similar to (40) and (41), when the harvested energy can afford all the $N$ UL data streams, the SAP will consume $N-1$ or $N$ units energy depending on the energy arrivals in next subframe, and we have

$$
p_{k, k-(N-1)}=\nu\left(1-q_{\mathrm{D}}\right)\left(1-p_{\mathrm{u}, \mathrm{void}}\right), N \leq k \leq K,
$$

$$
p_{k, k-N}=(1-\nu)\left(1-q_{\mathrm{D}}\right)\left(1-p_{\mathrm{u}, \mathrm{void}}\right), N \leq k \leq K,
$$

With the assumption that the rechargeable battery is available for DL transmissions as long as the harvested energy can support one DL data stream, we have the following transition probabilities based on the amount of harvested energy.

$$
p_{k, k-(n \omega-1)}= \begin{cases}\nu q_{\mathrm{D}}\left(1-p_{\mathrm{s}, \text { void }}\right), & n \in\{1,2, \ldots, N-1\}, \\ & n \omega-1 \leq k \leq(n+1) \omega-2, \\ \nu q_{\mathrm{D}}\left(1-p_{\mathrm{s}, \text { void }}\right), & n=N, \omega N-1 \leq k \leq K,\end{cases}
$$

$$
p_{k, k-n \omega}= \begin{cases}(1-\nu) q_{\mathrm{D}}\left(1-p_{\mathrm{s}, \text { void }}\right), & n \in\{1,2, \ldots, N-1\}, \\ & n \omega \leq k \leq(n+1) \omega-1 \\ (1-\nu) q_{\mathrm{D}}\left(1-p_{\mathrm{s}, \text { void }}\right), & \omega N \leq k \leq K\end{cases}
$$

Where the second equation in the right hand of (49) and (50) shows that when the harvested energy is able to simultaneously afford all the $N$ DL data streams, it will always serve $N$ DL data streams.

\section{B. Proof of Lemma 2}

Given the typical link length $r_{0}>0$, the Laplace transform of interference $\mathcal{L}_{I_{\mathrm{D} \rightarrow \mathrm{D}}}(s)=\mathbb{E}_{I_{\mathrm{D} \rightarrow \mathrm{D}}}\left[e^{-s I_{\mathrm{D} \rightarrow \mathrm{D}}}\right]$ can be derived as

$$
\begin{aligned}
& \mathbb{E}_{I_{\mathrm{D} \rightarrow \mathrm{D}}}\left[e^{\left.-s I_{\mathrm{D} \rightarrow \mathrm{D}}\right]}\right. \\
\stackrel{(a)}{=} & \exp \left(-\lambda_{\mathrm{S}, \mathrm{D}} \int_{\mathbb{R}^{2}}\left(1-\mathcal{L}_{g_{\mathbf{x}_{i}, \mathrm{SAP}}^{\mathrm{D}}}\left(s P_{\mathrm{S}} x^{-\alpha}\right)\right) \mathrm{d} x\right) \\
\stackrel{(b)}{=} & \exp \left(-\lambda_{\mathrm{s}, \mathrm{D}} \int_{\mathbb{R}^{2}}\left(1-\frac{1}{\left(1+s P_{\mathrm{S}} x^{-\alpha}\right)^{N}}\right) \mathrm{d} x\right) \\
\stackrel{(c)}{=} & \exp \left(-2 \pi \lambda_{\mathrm{s}, \mathrm{D}} \sum_{k=1}^{N}\left(\begin{array}{l}
N \\
k
\end{array}\right) \int_{0}^{\infty} \frac{\left(s P_{\mathrm{s}} r^{-\alpha}\right)^{k}}{\left(1+s P_{\mathrm{s}} r^{-\alpha}\right)^{N}} r \mathrm{~d} r\right) \\
\stackrel{(d)}{=} & \exp \left(-2 \pi \frac{\lambda_{\mathrm{s}, \mathrm{D}}}{\alpha}\left(s P_{\mathrm{S}}\right)^{\frac{2}{\alpha}}\right. \\
& \left.\cdot \sum_{k=1}^{N}\left(\begin{array}{l}
N \\
k
\end{array}\right) \int_{0}^{1} u^{N-k+\frac{2}{\alpha}-1}(1-u)^{k-\frac{2}{\alpha}-1} \mathrm{~d} u\right) \\
\stackrel{(e)}{=} & \exp \left(-2 \pi \frac{\lambda_{\mathrm{s}, \mathrm{D}}}{\alpha}\left(s P_{\mathrm{S}}\right)^{\frac{2}{\alpha}} \sum_{k=1}^{N}\left(\begin{array}{l}
N \\
k
\end{array}\right)\right. \\
&
\end{aligned}
$$

where (a) follows from the probability generating functional (PGFL) of PPP [43], (b) follows from the Laplace transform of the $g_{\mathbf{x}_{i}, \mathrm{SAP}}^{\mathrm{D}} \sim \Gamma(N, 1)$, (c) follows from Binomial theorem and the change from Cartesian to polar coordinates, (d) follows by first substituting $\left(s P_{\mathrm{S}}\right)^{-\frac{1}{\alpha}} r \rightarrow t$, then $\left(1+t^{-\alpha}\right)^{-1} \rightarrow u$, and (e) is due to the incomplete Beta function $B(a, b)=$ $\int_{0}^{1} u^{a-1}(1-u)^{b-1} d u$. Define $\mathcal{C}(\alpha, N)=\frac{2}{\alpha} \sum_{k=1}^{N}\left(\begin{array}{c}N \\ k\end{array}\right) \cdot B(N-$ $\left.k+\frac{2}{\alpha}, k-\frac{2}{\alpha}\right)$, and with $\lambda_{\mathrm{s}, \mathrm{D}}=q_{\mathrm{D}} \lambda_{\mathrm{s}}\left(1-p_{\mathrm{s}, \text { void }}\right)$, we have $\mathbb{E}_{I_{\mathrm{D} \rightarrow \mathrm{D}}}\left[e^{-s I_{\mathrm{D} \rightarrow \mathrm{D}}}\right]=\exp \left(-\pi q_{\mathrm{D}} \lambda_{\mathrm{s}}\left(1-p_{\mathrm{s}, \text { void }}\right)\left(s P_{\mathrm{s}}\right)^{\frac{2}{\alpha}} \mathcal{C}(\alpha, N)\right)$.

The derivation of Laplace transform of interference $\mathcal{L}_{I_{\mathrm{D} \rightarrow \mathrm{U}}}(s)=\mathbb{E}_{I_{\mathrm{D} \rightarrow \mathrm{U}}}\left[e^{-s I_{\mathrm{D} \rightarrow \mathrm{U}}}\right]$ is similar to that of $\mathcal{L}_{I_{\mathrm{D} \rightarrow \mathrm{D}}}(s)$. Thus, we omit the steps and directly give the result

$\mathbb{E}_{I_{\mathrm{D} \rightarrow \mathrm{U}}}\left[e^{-s I_{\mathrm{D} \rightarrow \mathrm{U}}}\right]=\exp \left(-\pi q_{\mathrm{D}} \lambda_{\mathrm{s}}\left(1-p_{\mathrm{s}, \text { void }}\right)\left(s P_{\mathrm{s}}\right)^{\frac{2}{\alpha}} \mathcal{C}(\alpha, N)\right)$.

For $I_{\mathrm{U} \rightarrow \mathrm{D}}$, we observe that the spatial locations of UL MUs $\Phi_{\mathrm{u}}^{\mathrm{t}}$ form a cluster point process of intensity $\lambda_{\mathrm{u}, \mathrm{t}}=(1-$ $\left.q_{\mathrm{D}}\right) N_{\mathrm{a}} \lambda_{\mathrm{s}}$. However, the exact analysis of cluster point process leads to tedious expressions of the interference distribution, making it difficult to derive the insight of interferers. We approximate the UL MUs by a PPP with the same intensity $\lambda_{\mathrm{u}, \mathrm{t}}$, which leads to a lower bound on the coverage probability [44]. The Laplace transform of interference $I_{\mathrm{U} \rightarrow \mathrm{D}}$ and $I_{\mathrm{U} \rightarrow \mathrm{U}}$ can be derived by using the PGFL of the PPP, given by

$$
\begin{aligned}
& \begin{aligned}
\mathbb{E}_{I_{\mathrm{U} \rightarrow \mathrm{D}}}\left[e^{\left.-s I_{\mathrm{U} \rightarrow \mathrm{D}}\right]}\right. & =\mathbb{E}_{I_{\mathrm{U} \rightarrow \mathrm{U}}}\left[e^{-s I_{\mathrm{U} \rightarrow \mathrm{U}}}\right] \\
& \simeq \exp \left(-\pi\left(1-q_{\mathrm{D}}\right) N_{\mathrm{a}} \lambda_{\mathrm{s}}\left(s Q_{\mathrm{u}}\right)^{\frac{2}{\alpha}} \delta(\alpha)\right),
\end{aligned} \\
& \text { where } \delta(\alpha) \triangleq \frac{2 \pi / \alpha}{\sin (2 \pi / \alpha)} \\
& \quad \text { C. Proof of Theorem } 1
\end{aligned}
$$

By definition, the DL coverage can be derived as

$$
\begin{aligned}
\mathbb{P}_{\mathrm{D}} & =\operatorname{Pr}\left(\operatorname{SINR}_{\mathrm{D}}>\gamma_{\mathrm{D}}\right) \\
& =\mathbb{E}\left[\operatorname{Pr}\left(h_{0,0}^{\mathrm{D}}>\frac{\gamma_{\mathrm{D}}\left(I_{\mathrm{D} \rightarrow \mathrm{D}}+I_{\mathrm{U} \rightarrow \mathrm{D}}+\sigma^{2}\right)}{P_{\mathrm{s}} r_{0}^{-\alpha}}\right)\right] \\
& \left.\stackrel{(a)}{=} \int_{0}^{\infty} \operatorname{Pr}\left(h_{0,0}^{\mathrm{D}}>s I_{I N}\right) f_{R_{\mathrm{cl}}}\left(r_{0}\right) \mathrm{d} r_{0}\right]
\end{aligned}
$$


where in (a) we define $s=\frac{\gamma_{\mathrm{D}} r_{0}^{\alpha}}{P_{\mathrm{s}}}$ and $I_{I N}=I_{\mathrm{D} \rightarrow \mathrm{D}}+I_{\mathrm{U} \rightarrow \mathrm{D}}+\sigma^{2}$. The probability distribution function (PDF) of the typical link length is

$$
f_{R_{\mathrm{cl}}}\left(r_{0}\right)=\left\{\begin{array}{ll}
\frac{2 r_{0}}{R_{\mathrm{cl}}^{2}}, & r_{0} \in\left[0, R_{\mathrm{cl}}\right] \\
0, & \text { otherwise }
\end{array} .\right.
$$

Letting $\mathbb{P}_{\mathrm{D}}\left(r_{0}\right)=\operatorname{Pr}\left(h_{0,0}^{\mathrm{D}}>s I_{I N}\right)$, the conditional coverage probability is given by

$$
\begin{aligned}
& \mathbb{P}_{\mathrm{D}}\left(r_{0}\right) \\
& =\quad \operatorname{Pr}\left(h_{0,0}^{\mathrm{D}}>s I_{I N}\right) \stackrel{(a)}{=} \sum_{i=0}^{M-N} \frac{1}{i !} \mathbb{E}_{I_{I N}}\left[\left(s I_{I N}\right)^{i} e^{-s I_{I N}}\right] \\
& \stackrel{(b)}{=} \quad \sum_{i=0}^{M-N} \frac{1}{i !}(-s)^{i} \frac{d^{i}}{d s^{i}} \mathcal{L}_{I_{I N}}(s)
\end{aligned}
$$

where (a) follows from the CCDF of a Gamma variable $X \sim$ $\Gamma(k, \theta)$, and (b) is derived by substituting $\mathbb{E}_{X}\left[X^{n} e^{-s X}\right]=$ $(-1)^{n} \frac{\mathrm{d}^{n}}{\mathrm{~d} s^{n}} \mathcal{L}_{X}(s)$ and $h_{0,0}^{\mathrm{D}} \sim \Gamma(M-N+1,1)$.

To derive $\mathbb{P}_{\mathrm{D}}\left(r_{0}\right)$, we need to first compute the $i$ th derivative of the Laplace transform of $I_{I N}$.

$$
\begin{aligned}
\mathcal{L}_{I_{I N}}(s) & =\mathbb{E}\left[e^{-s \sigma^{2}}\right] \mathbb{E}_{I_{\mathrm{D} \rightarrow \mathrm{D}}}\left[e^{-s I_{\mathrm{D} \rightarrow \mathrm{D}}}\right] \mathbb{E}_{I_{\mathrm{U} \rightarrow \mathrm{D}}}\left[e^{-s I_{\mathrm{U} \rightarrow \mathrm{D}}}\right] \\
& =\exp \left(-s \sigma^{2}-\pi q_{\mathrm{D}} \lambda_{\mathrm{S}}\left(1-p_{\mathrm{s}, \mathrm{void}}\right)\left(s P_{\mathrm{s}}\right)^{\frac{2}{\alpha}} \mathcal{C}(\alpha, N)\right. \\
& \left.-\pi\left(1-q_{\mathrm{D}}\right) N_{\mathrm{a}} \lambda_{\mathrm{S}}\left(s Q_{\mathrm{u}}\right)^{\frac{2}{\alpha}} \delta(\alpha)\right) .
\end{aligned}
$$

Letting $f(g(s))=\mathcal{L}_{I_{I N}}(s)$ with $f(x)=\exp (x)$, i.e.,

$$
\begin{aligned}
g(s)= & -s \sigma^{2}-\pi q_{\mathrm{D}} \lambda_{\mathrm{s}}\left(1-p_{\mathrm{s}, \text { void }}\right)\left(s P_{\mathrm{s}}\right)^{\frac{2}{\alpha}} \mathcal{C}(\alpha, N) \\
& -\pi\left(1-q_{\mathrm{D}}\right) N_{\mathrm{a}} \lambda_{\mathrm{s}}\left(s Q_{\mathrm{u}}\right)^{\frac{2}{\alpha}} \delta(\alpha) .
\end{aligned}
$$

Using the Faà di Bruno's formula, we derive the $i$ th derivative of Laplace transform of $I_{I N}$ as [11]

$$
\begin{aligned}
\frac{\mathrm{d}^{i} \mathcal{L}_{I_{I N}}(s)}{\mathrm{d} s^{i}} & =\frac{\mathrm{d}^{i} f(g(s))}{\mathrm{d} s^{i}} \\
& =\sum \frac{i !}{j_{1} ! j_{2} ! \ldots j_{i} !} f^{\left(\sum_{k=1}^{i} j_{k}\right)}(g(s)) \prod_{l=1}^{i}\left(\frac{g^{l}(s)}{l !}\right)^{j_{l}}
\end{aligned}
$$

The $l$ th derivative of $g(s)$ is given by

$$
\begin{aligned}
g^{l}(s)= & -\sigma^{2} 1_{(l=1)}-\prod_{n=0}^{l-1}\left(\frac{2}{\alpha}-n\right) s^{\frac{2}{\alpha}-l}\left[\pi q_{\mathrm{D}} \lambda_{\mathrm{s}}\left(1-p_{\mathrm{s}, \text { void }}\right)\right. \\
& \left.\cdot P_{\mathrm{s}}^{\frac{2}{\alpha}} \mathcal{C}(\alpha, N)-\pi\left(1-q_{\mathrm{D}}\right) N_{\mathrm{a}} \lambda_{\mathrm{s}} Q_{\mathrm{u}}^{\frac{2}{\alpha}} \delta(\alpha)\right]
\end{aligned}
$$

where $1_{(\cdot)}$ is the indicator function. Since $\sum_{k=1}^{i} j_{k} \in \mathbb{N}$ and $f(x)=\exp (x)$, we have $f^{\left(\sum_{k=1}^{i} j_{k}\right)}(g(s))=\mathcal{L}_{I_{I N}}(s)$.

Combining (53)-(56), we derive the conditional coverage probability as

$$
\mathbb{P}_{\mathrm{D}}\left(r_{0}\right)=\sum_{i=0}^{M-N} \frac{(-s)^{i}}{i !} \mathcal{L}_{I_{I N}}(s) \sum \frac{i !}{j_{1} ! j_{2} ! \ldots j_{i} !} \prod_{l=1}^{i}\left(\frac{g^{l}(s)}{l !}\right)^{j_{l}} .
$$

The DL coverage probability is derived by substituting (52) and (57) into (51), given by

$$
\begin{aligned}
\mathbb{P}_{\mathrm{D}}=\int_{0}^{R_{\mathrm{cl}}} & \sum_{i=0}^{M-N} \frac{(-s)^{i}}{i !} \mathcal{L}_{I_{I N}}(s) \\
& \cdot \sum \frac{i !}{j_{1} ! j_{2} ! \ldots j_{i} !} \prod_{l=1}^{i}\left(\frac{g^{l}(s)}{l !}\right)^{j_{l}} \frac{2 r_{0}}{R_{\mathrm{cl}}^{2}} \mathrm{~d} r_{0} .
\end{aligned}
$$

The coverage probability of the typical MU associated with the tagged UL SAP is given by

$$
\mathbb{P}_{\mathrm{U}}=\int_{0}^{\infty} \operatorname{Pr}\left(h_{0,0}^{\mathrm{U}}>s I_{I N}\right) f_{R_{\mathrm{cl}}}\left(r_{0}\right) \mathrm{d} r_{0},
$$

where $h_{0,0}^{\mathrm{U}} \sim \Gamma\left(M-N_{\mathrm{a}}+1,1\right), s=\frac{\gamma_{\mathrm{U}} r_{0}^{\alpha}}{Q_{\mathrm{u}}}$, and $I_{I N}=$ $I_{\mathrm{D} \rightarrow \mathrm{D}}+I_{\mathrm{U} \rightarrow \mathrm{D}}+\sigma^{2}$. Similar to the derivation of $\mathbb{P}_{\mathrm{D}}$, we derive the UL coverage probability as

$$
\begin{aligned}
\mathbb{P}_{\mathrm{U}}=\int_{0}^{R_{\mathrm{cl}}} & \sum_{i=0}^{M-N_{\mathrm{a}}} \frac{1}{i !}(-s)^{i} \mathcal{L}_{I_{I N}}(s) \\
& \cdot \sum \frac{i !}{j_{1} ! j_{2} ! \ldots j_{i} !} \prod_{l=1}^{i}\left(\frac{g^{l}(s)}{l !}\right)^{j_{l}} \frac{2 r_{0}}{R_{\mathrm{cl}}^{2}} \mathrm{~d} r_{0} .
\end{aligned}
$$

\section{REFERENCES}

[1] Cisco, "Cisco visual networking index: Global mobile data traffic forecast update, 2011-2016," white paper, Feb. 2012.

[2] T. Q. S. Quek, G. de la Roche, I. Guvenc, and M. Kountouris, Small Cell Networks: Deployment, PHY Techniques, and Resource Management. New York, NY, USA: Cambridge Univ. Press, 2013.

[3] Q. Li, G. Li, W. Lee, M. il Lee, D. Mazzarese, B. Clerckx, and Z. Li, "MIMO techniques in WiMAX and LTE: A feature overview," IEEE Commun. Mag., vol. 48, no. 5, pp. 86-92, May 2010.

[4] J. G. Andrews, "Seven ways that HetNets are a cellular paradigm shift," IEEE Commun. Mag., vol. 51, no. 3, pp. 136-144, Mar. 2013.

[5] J. Mecke, Stochastic geometry and its applications. Chichester: Wiley, 1995.

[6] J. G. Andrews, F. Baccelli, and R. Ganti, "A tractable approach to coverage and rate in cellular networks," IEEE Trans. Commun., vol. 59, no. 11 , pp. 3122-3134, Nov. 2011.

[7] S. Singh, H. S. Dhillon, and J. G. Andrews, "Offloading in heterogeneous networks: Modeling, analysis, and design insights," IEEE Trans. Wireless Commun., vol. 12, no. 5, pp. 2484-2497, May 2013.

[8] W. C. Cheung, T. Q. S. Quek, and M. Kountouris, "Throughput optimization, spectrum allocation, and access control in two-tier femtocell networks," IEEE J. Sel. Areas Commun., vol. 30, no. 3, pp. 561-574, April 2012.

[9] V. Chandrasekhar, M. Kountouris, and J. G. Andrews, "Coverage in multi-antenna two-tier networks," IEEE Trans. Wireless Commun., vol. 8, no. 10, pp. 5314-5327, October 2009.

[10] H. Dhillon, M. Kountouris, and J. G. Andrews, "Downlink MIMO HetNets: Modeling, ordering results and performance analysis," IEEE Trans. Wireless Commun., vol. 12, no. 10, pp. 5208-5222, Oct. 2013.

[11] A. Gupta, H. Dhillon, S. Vishwanath, and J. G. Andrews, "Downlink multi-antenna heterogeneous cellular network with load balancing," IEEE Trans. Commun., vol. 62, no. 11, pp. 4052-4067, Nov. 2014.

[12] S. Sesia, I. Toufik, and M. Baker, LTE- The UMTS Long Term Evolution: From Theory to Practice. New Jersey, NJ, USA: John Wiley \& Sons, 2011.

[13] 3GPP TR 36.828, V11.0.0, Technical Specification Group Radio Access Network; Further Enhancements to LTE TDD for DL-UL Interference Management and Traffic Adaptation (Release 11), 3GPP Std., June 2012.

[14] Draft Report of 3GPP TSG RAN WG1 \#77, ETSI MCC Std., May 2014.

[15] Z. Shen, A. Khoryaev, E. Eriksson, and X. Pan, "Dynamic uplinkdownlink configuration and interference management in TD-LTE," IEEE Commun. Mag., vol. 50, no. 11, pp. 51-59, Nov. 2012.

[16] A. Khoryaev, M. Chervyakov, M. Shilov, S. Panteleev, and A. Lomayev, "Performance analysis of dynamic adjustment of TDD uplink-downlink configurations in outdoor picocell LTE networks," in Proc. ICUMT workshops, St. Petersburg, Oct. 3-5, 2012, pp. 914-921. 
[17] Y. S. Soh, T. Q. S. Quek, M. Kountouris, and G. Caire, "Cognitive hybrid division duplex for two-tier femtocell networks," IEEE Trans. Wireless Commun., vol. 12, no. 10, pp. 4852-4865, Oct. 2013.

[18] B. Yu, S. Mukherjee, H. Ishii, and L. Yang, "Dynamic TDD support in the LTE-B enhanced local area architecture," in IEEE GLOBECOM Workshops, Anaheim, America, Dec. 3-7, 2012, pp. 585-591.

[19] H. Sun, M. Sheng, M. Wildemeersch, and T. Q. S. Quek, "Coverage analysis for two-tier dynamic TDD heterogeneous networks," in Proc. IEEE GLOBECOM, Austin, TX, Dec. 8-12, 2014, pp. 3672-3677.

[20] A. Fehske, G. Fettweis, J. Malmodin, and G. Biczok, "The global footprint of mobile communicatons: The ecological and economic perspective," IEEE Commun. Mag., vol. 49, no. 8, pp. 55-62,, Aug. 2011.

[21] I. Ashraf, F. Boccardi, and L. Ho, "SLEEP mode techniques for small cell deployments," IEEE Commun. Mag., vol. 49, no. 8, pp. 72-79, Aug. 2011.

[22] M. Wildemeersch, T. Q. S. Quek, C. Slump, and A. Rabbachin, "Cognitive small cell networks: Energy efficiency and trade-offs," IEEE Trans. Commun., vol. 61, no. 9, pp. 4016-4029, Sept. 2013.

[23] Y. S. Soh, T. Q. S. Quek, M. Kountouris, and H. Shin, "Energy efficient heterogeneous cellular networks," IEEE J. Sel. Areas Commun., vol. 31 , no. 5, pp. 840-850, May 2013.

[24] H. Dhillon, Y. Li, P. Nuggehalli, Z. Pi, and J. G. Andrews, "Fundamentals of heterogeneous cellular networks with energy harvesting," IEEE Trans. Wireless Commun., vol. 13, no. 5, pp. 2782-2797, April 2014.

[25] P.-S. Yu, J. Lee, T. Q. S. Quek, and Y.-W. P. Hong, "Traffic offloading in heterogeneous networks with energy harvesting personal cells - Network throughput and energy efficiency," IEEE Trans. Wireless Commun., vol. 15, no. 2, pp. 1146-1161, Feb. 2016.

[26] H. Yang, J. Lee, and T. Q. S. Quek, "Heterogeneous network with energy harvesting based D2D communication," IEEE Trans. Wireless Commun., vol. 15, no. 2, pp. 1406-1419, Feb. 2016.

[27] J. Paradiso and T. Starner, "Energy scavenging for mobile and wireless electronics," IEEE Pervasive Comput., vol. 4, no. 1, pp. 18-27, Jan. 2005.

[28] O. Ozel, K. Tutuncuoglu, J. Yang, S. Ulukus, and A. Yener, "Transmission with energy harvesting nodes in fading wireless channels: Optimal policies," IEEE J. Sel. Areas Commun., vol. 29, no. 8, pp. 1732-1743, Sept. 2011.

[29] O. Ozel, J. Yang, and S. Ulukus, "Optimal broadcast scheduling for an energy harvesting rechargeable transmitter with a finite capacity battery," IEEE Trans. Wireless Commun., vol. 11, no. 6, pp. 2193-2203, June 2012.

[30] J. Yang and S. Ulukus, "Optimal packet scheduling in an energy harvesting communication system," IEEE Trans. Commun., vol. 60, no. 1, pp. 220-230, Jan. 2012.

[31] M. Antepli, E. Uysal-Biyikoglu, and H. Erkal, "Optimal packet scheduling on an energy harvesting broadcast link," IEEE J. Sel. Areas Commun., vol. 29, no. 8, pp. 1721-1731, Sept. 2011.

[32] M. S. ElBamby, M. Bennis, W. Saad, and M. Latva-aho, "Dynamic uplink-downlink optimization in TDD-based small cell networks," in Proc. IEEE INFOCOM Workshops, Toronto, Canada, Apr. 27 - May 2, 2014, pp. 712-717.

[33] D. Gross, J. F. Shortle, and C. M. Harris, Fundamentals of Queueing Theory, 4th ed. Hoboken, NJ: John Wiley \& Sons, 2008.

[34] R. Ganti and M. Haenggi, "Spatial and temporal correlation of the interference in aloha ad hoc networks," IEEE Commun. Lett., vol. 13, no. 9, pp. 631-633, Sept. 2009.

[35] J. Zhang, M. Kountouris, J. G. Andrews, and R. W. Heath, "Multi-mode transmission for the MIMO broadcast channel with imperfect channel state information," IEEE Trans. Commun., vol. 59, no. 3, pp. 803-814, March 2011.

[36] J. Liu, M. Sheng, T. Q. S. Quek, and J. Li, "D2D Enhanced Co-Ordinated Multipoint in Cloud Radio Access Networks," IEEE Trans. Wireless Commun., vol. 15, no. 6, pp. 4248-4262, Mar. 2016.

[37] M. Haenggi, "Local delay in static and highly mobile poisson networks with aloha," in Proc. IEEE ICC, Cape Town, May 23-27 2010, pp. 1-5.

[38] P. Wu and N. Jindal, "Coding versus ARQ in fading channels: How reliable should the PHY be?" IEEE Trans. Commun., vol. 59, no. 12, pp. 3363-3374, Dec. 2011.

[39] D. Nguyen, L.-N. Tran, P. Pirinen, and M. Latva-aho, "Precoding for full duplex multiuser MIMO systems: Spectral and energy efficiency maximization," IEEE Trans. Signal Process., vol. 61, no. 16, pp. 4038 4050, Aug. 2013.

[40] J. Jeon and A. Ephremides, "The stability region of random multiple access under stochastic energy harvesting," in Proc. IEEE Int. Symp. on Inf. Theory (ISIT), July 2011, pp. 1796-1800.
[41] Y. Cui, V. Lau, and Y. Wu, "Delay-aware BS discontinuous transmission control and user scheduling for energy harvesting downlink coordinated MIMO systems," IEEE Trans. Signal Process., vol. 60, no. 7, pp. 37863795, July 2012.

[42] N. Jindal, J. G. Andrews, and S. Weber, "Multi-antenna communication in ad hoc networks: Achieving MIMO gains with SIMO transmission," IEEE Trans. Commun., vol. 59, no. 2, pp. 529-540, February 2011.

[43] M. Haenggi, Stochastic Geometry for Wireless Networks. Combridge University Press, 2012.

[44] R. Ganti and M. Haenggi, "Interference and outage in clustered wireless ad hoc networks," IEEE Trans. Inf. Theory, vol. 55, no. 9, pp. 40674086, Sept 2009.

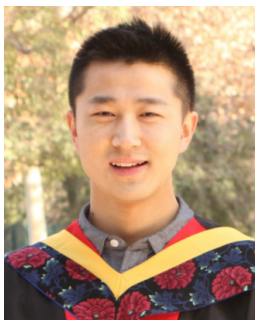

Hongguang Sun (S'14-M'16) received the B.S degree (with distinction) in Electronic and Information Engineering from Northeastern University, Qinhuangdao, China, in 2009 and the Ph.D. degree in Communication and Information System from Xidian University, Xi'an, China, in December 2015. From January to July 2015, he was a Visiting Student at the Singapore University of Technology and Design, Singapore. Currently, he is a Lecturer with the School of Telecommunications Engineering, Xidian University. His research interests include dynamic resource management, D2D communications, ultra-dense networks, and performance analysis in heterogeneous wireless networks, applying tools from probability theory and stochastic geometry. Dr. Sun served as a Technical Program Committee member for the Wireless Communications Systems Symposium for IEEE/CIC ICCC 2016, and for the Mobile Network Applications and Services Track for IEEE VTC 2017-Spring.

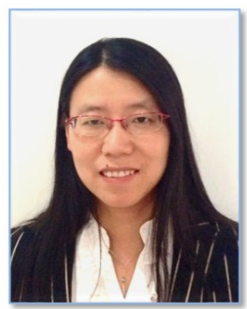

Min Sheng (M'03-SM'16) received the M.S. and $\mathrm{Ph} . \mathrm{D}$. degrees in communication and information systems from Xidian University, Shaanxi, China, in 2000 and 2004, respectively. She has been a faculty member of the School of Telecommunications Engineering at Xidian University since 2000, where she is currently a Full professor with the State Key Laboratory of ISN. Her current research interests include interference and resource management in heterogeneous networks, ultra dense networks (UDN), self-organizing networks (SON), big data processing, green communications, and satellite networks. She has published 2 books and over 130 papers in refereed journals and conference proceedings. She was honored with the Second Prize for the State Technological Innovation Award in 2014, the New Century Excellent Talents in University by the Ministry of Education of China, the Young Teachers Award from the Fok Ying-Tong Education Foundation, China, in 2008, and the Best Paper Award at IEEE/CIC ICCC 2013. 


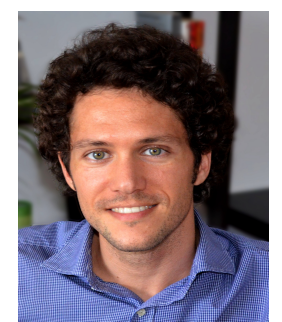

Matthias Wildemeersch (S'09-M'13) received the M.Sc. degree in electromechanical engineering from the University of Ghent, Belgium, and the Ph.D. degree in electrical engineering at the University of Twente, The Netherlands. He gained professional experience at the Joint Research Centre of the European Commission, the Agency for Science, Technology and Research $\left(A^{\star}\right.$ STAR) in Singapore, and the Singapore University of Technology and Design (SUTD). Currently, he is a research scholar at the International Institute for Applied Systems Analysis (IIASA), where his work broadly covers the dynamical behavior of large-scale, multi-agent networks. His research interests span various aspects of signal processing and network dynamics, applying tools from probability theory and control. He received the IEEE SPAWC 2013 Best Student Paper Award and he is an awardee of the A*STAR Research Attachment Program (2012-2013) and the IIASA Postdoctoral Fellowship (2015-2016).

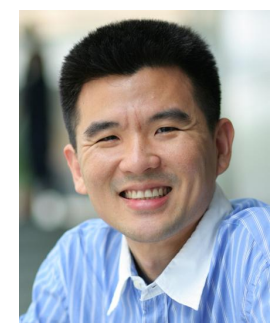

Tony Q. S. Quek (S'98-M'08-SM'12) received the B.E. and M.E. degrees in Electrical and Electronics Engineering from Tokyo Institute of Technology, Tokyo, Japan, respectively. At Massachusetts Institute of Technology, he earned the Ph.D. in Electrical Engineering and Computer Science. Currently, he is an Assistant Professor with the Information Systems Technology and Design Pillar at Singapore University of Technology and Design (SUTD). He is also a deputy director of SUTD-ZJU IDEA. His main research interests are the application of mathematical, optimization, and statistical theories to communication, networking, signal processing, and resource allocation problems. Specific current research topics include heterogeneous networks, green communications, wireless security, IoT, big data processing, and cognitive radio.

He has been actively involved in organizing and chairing sessions, and has served as a Member of the Technical Program Committee as well as Symposium Chair in a number of international conferences. He is a coauthor of the book Small Cell Networks: Deployment, PHY Techniques, and Resource Allocation (Cambridge University Press, 2013) and the book Cloud Radio Access Networks: Principles, Technologies, and Applications (Cambridge University Press, 2016). He is currently an Editor for the IEEE TRANSACTIONS ON COMMUNICATIONS and an Executive Editorial Committee Member for the IEEE TRANSACTIONS ON WIRELESS COMMUNICATIONS. He was an Editor for the IEEE WIRELESS COMMUNICATIONS LETTERS, a Guest Editor for the IEEE Signal Processing Magazine (Special Issue on Signal Processing for the 5G Revolution) in 2014, and the IEEE Wireless Communications Magazine (Special Issue on Heterogeneous Cloud Radio Access Networks) in 2015.

Dr. Quek was the recipient of the 2008 Philip Yeo Prize for Outstanding Achievement in Research, the IEEE Globecom 2010 Best Paper Award, the CAS Fellowship for Young International Scientists in 2011, the 2012 IEEE William R. Bennett Prize, the IEEE SPAWC 2013 Best Student Paper Award, the IEEE WCSP 2014 Best Paper Award, the IEEE PES General Meeting 2015 Best Paper, and the 2015 SUTD Outstanding Education Awards-Excellence in Research.

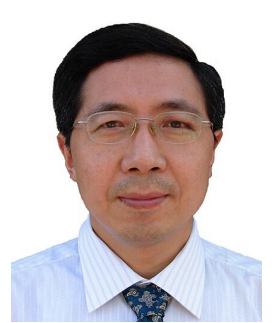

Jiandong Li (SM'05) received the B.E., M.S. and $\mathrm{Ph} . \mathrm{D}$. degrees in Communications Engineering from Xidian University, Xi' an, China, in 1982, 1985 and 1991 respectively. In 1985, he joined Xidian University, where he has been a Full Professor since 1994 and the Vice President since 2012. Dr. Li is a Fellow of the China Institute of Electronics and the China Institute of Communication. His major research interests include wireless communication theory, cognitive and software radio, self-organizing networks and signal processing. He was a visiting professor to the Department of Electrical and Computer Engineering at Cornell University from 2002-2003. He served as the General Vice Chair for ChinaCom 2009 and TPC Chair of IEEE ICCC 2013. He was awarded as the Distinguished Young Researcher from NSFC, Changjiang Scholar from Ministry of Education, China, and honored with the Second Prize for the State Technological Innovation Award in 2014. 